\title{
Materialia
}

Available online 16 May 2018

In Press, Corrected Proof ??

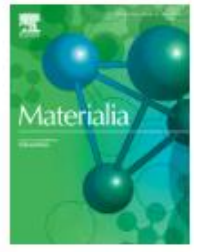

Full Length Article

\section{Influence of strain rate on dynamic transformation of austenite in an as-cast medium-carbon low-alloy steel}

K. Chadha ${ }^{a} \curvearrowleft{ }^{\otimes}$, Z. Ahmed $^{a}$, C. Aranas Jr. ${ }^{b}$, D. Shahriari ${ }^{a}$, M. Jahazi ${ }^{a} \circ{ }^{\circ}$

田 Show more

https://doi.org/10.1016/j.mtla.2018.04.006 


\title{
Influence of Strain Rate on Dynamic Transformation of Austenite in an As-Cast Medium-Carbon Low-Alloy Steel
}

\author{
K. Chadha ${ }^{1}$, Z. Ahmed ${ }^{1}$, C. Jr. Aranas ${ }^{2}$, D. Shahriari ${ }^{1}$, M. Jahazi ${ }^{1}$ \\ ${ }^{1}$ Department of Mechanical Engineering, École de technologie supérieure (ÉTS), \\ 1100 Notre Dame Street West, Montreal, Quebec, H3C 1K3, Canada \\ ${ }^{2}$ Department of Mechanical Engineering, University of New Brunswick \\ Fredericton, NB, E3B 5A3, Canada
}

\begin{abstract}
In this work, the effect of strain rate on the dynamic transformation (DT) of austenite to ferrite during high temperature forging of an as-cast medium-carbon low-alloy steel was investigated. Hot deformation experiments were carried out in the $1150{ }^{\circ} \mathrm{C}$ to $1200{ }^{\circ} \mathrm{C}$ temperature range (400$450{ }^{\circ} \mathrm{C}$ above $\mathrm{Ae}_{3}$ ) and $0.25 \mathrm{~s}^{-1}$ to $2 \mathrm{~s}^{-1}$ strain rate range using a Gleeble $3800^{\circledR}$ thermomechanical simulator. The critical strains for DT and dynamic recrystallization (DRX) were determined to be in the 0.08 to 0.18 strain range. A microstructural analysis was conducted using optical and electron microscopy. The kernel average misorientation (KAM) technique was applied to electron back-scattered diffraction (EBSD) images to quantify the internal misorientation of grains for the characterization of DT ferrite. Furthermore, it was found that an increase in strain rate decreased the amount of dynamically formed ferrite under the same applied strain and testing temperature. The obtained results were correlated with the influence of deformation parameters on carbon diffusivity and its impact on the growth of dynamically formed ferrite. It was found that an increase in the strain rate decreased the diffusion distance of carbon, which was the responsible mechanism for decreased ferrite formation at higher strain rates.
\end{abstract}

Keywords: Low-alloy steel, As-cast structure, Hot deformation, Dynamic transformation, Carbon diffusion, EBSD analysis.

\section{Introduction}

High-strength steels used for the manufacturing of large size components such as turbine shafts are produced by ingot casting, followed by forging, quenching, and tempering operations. The first step of the deformation process, called ingot breakdown, is carried out well above the paraequilibrium $\mathrm{Ae}_{3}$ temperature (temperature at which ferrite to austenite transformation 
accompanied by interstitial diffusion reaches equilibrium [1]), and therefore produces major microstructural changes, such as dynamic recovery (DRV), dynamic recrystallization (DRX) and metadynamic recrystallization (MDRX) [2-4]. Deformation-induced transformation of austenite to ferrite, also known as dynamic transformation (DT), is an additional softening mechanism that needs to be taken into account. This phenomenon was first reported by Matsumara et al. and Yada et al. in the late 1980s [5, 6], and has attracted considerable interest in recent years [7-9]. However, most studies on DT are associated with wrought structures, and particularly rolled materials [9, 10], and very limited data is available on the dynamic transformation of austenite in as-cast structures.

The occurrence and extent of DT depend on various parameters, such as alloy chemistry $[11,12]$ and grain size prior to deformation [13], as well as process parameters (temperature, strain and strain rate) $[8,14]$. The alloy composition significantly impacts the extent of DT. Ghosh et al. [11] studied the effect of carbon content on DT, and observed that at $0.15 \%$ to 0.40 wt. \% carbon the Widmanstätten ferrite forms instantly from deformed austenite, whereas at a carbon content of $0.79 \mathrm{wt} . \%$, the formation of ferrite was accompanied by that of cementite. Aranas et al. [12] investigated the effect of Mn and Si on the occurrence of DT and reported that the presence of $\mathrm{Mn}$ increases not only the driving force, but also the thermodynamic energy barrier (i.e., the Gibbs energy difference between austenite and ferrite) for the occurrence of DT. On the other hand, Si increased the driving force, but decreased the thermodynamic energy barrier, thus proving to accelerate the DT process. N. Park et al. [13] observed that with a decrease in austenite grain size, the critical strain value decreased as well, implying that the onset of DT was accelerated. The effect of temperature and strain on DT was studied by Grewal et al. [14], who observed that as the temperature increased beyond the obstacle peak, it became easier to form Widmanstätten plates. In another study, N. Park et al. [8] observed that under slow strain rate conditions, higher dynamic transformation was observed; however, their study was not focused on the effect of strain rate and its related mechanisms on DT.

The occurrence of DT was initially related to the influence of deformation on the stored energy of dislocations [11]. However, it was later shown that the dislocations cannot accurately explain the dynamic transformation taking place well above the $\mathrm{Ae}_{3}$ temperature. As a result, a new model known as transformation softening was proposed by Jonas et al. [15]. In this model, the driving force for DT is the net softening during the phase change, which is defined by the flow 
stress difference between the strain-hardened austenite and the yield stress of the freshly formed Widmanstätten ferrite [15]. Upon isothermal holding, DT ferrite can retransform back to austenite, the thermodynamically stable phase $[12,16]$. However, among the existing proposed mechanisms for DT, less attention has been paid to the influence of strain rate on carbon diffusivity and its effect on DT. For instance, the nucleation and growth of ferrite plates are also related to the diffusion of alloying elements such as carbon [17]. Furthermore, deformation under low strain rates means the specimen is under compression stress longer time than at higher strain rates. As a result, the evolution of the nucleated ferrite at critical strain will depend on the strain rate [18-20].

The objectives of the present work are, therefore: i) to study dynamic transformation of austenite to ferrite in an as-cast structure, ii) to investigate and quantify the influence of strain rate on the nucleation and growth of the newly formed DT ferrite, and iii) to propose a mechanism which takes into account the role of solute (carbon) diffusion under various strain rates and its impact on the nucleation of DT ferrite.

\section{Materials and Methods}

The detailed composition of the as-cast medium-carbon low-alloy steel used for the experiments is shown in Table 1, along with its orthoequilibrium and paraequilibrium temperatures. These were calculated using the FactSage thermodynamic software, employing the FSStel Database ${ }^{\circledR}$ [21]. The materials were provided by Finkl Steel-Sorel Forge, Sorel, Quebec, Canada. Cylindrical specimens were machined from the central region of the as-cast ingot with diameters and heights of $10 \mathrm{~mm}$ and $15 \mathrm{~mm}$, respectively. Hot compression tests were carried out using the Gleeble $3800^{\circledR}$ thermomechanical simulator following the procedures described in the ASTM E209. The schematic diagram of the thermomechanical cycle is shown in Fig. 1. Two

temperatures, $1150{ }^{\circ} \mathrm{C}$ and $1200{ }^{\circ} \mathrm{C}$, and three strain rates, $0.25 \mathrm{~s}^{-1}, 1 \mathrm{~s}^{-1}$ and $2 \mathrm{~s}^{-1}$, were employed in the experiments. For reference, deformation temperatures were selected based on the actual parameters used during industrial ingot forging, which were measured using a FLIR ${ }^{\circledR}$ T650sc thermal camera. The thermomechanical procedure involved heating the sample to $1260{ }^{\circ} \mathrm{C}$ at a $2{ }^{\circ} \mathrm{C} / \mathrm{s}$ heating rate and soaking for 5 minutes (300 s) to homogenize the temperature. The samples were then cooled to the deformation temperature at a rate of $1{ }^{\circ} \mathrm{C} / \mathrm{s}$ before being compressed to a true strain of 0.8 , followed by water quenching [22]. $0.1 \mathrm{~mm}$ thick tantalum sheets were used as a lubricant between the sample and the pure tungsten anvils. The samples were polished 
mechanically using conventional techniques and then electropolished at room temperature using a mixture of Perchloric acid and ethanol (1:9 by volume) to reveal the microstructure by means of EBSD techniques. The electropolishing was conducted at $25 \mathrm{~V}$ for $15 \mathrm{~s}$. The samples were placed with their compression axis parallel to the incident electron beam. The EBSD analysis was performed using FEG-SEM (Carl Zeiss; Model: Supra 40) equipped with TSL-OIM ${ }^{\mathrm{TM}}$ software. Fig. 2 presents the initial microstructure of the as-cast material before homogenization. The microstructure shows a coarse grain structure with no specific grain boundaries. In order to observe the microstructure of the material just before deformation, i.e., in homogenized condition, one sample was subjected to homogenization at $1260{ }^{\circ} \mathrm{C}$ for 5 minutes, followed by water quenching. The homogenized grain boundary (G.B.) map of this microstructure is presented in Fig. 3, and shows very large grains. The low angle grain boundaries (LAGBs $\theta<2^{\circ}$ ) are marked with red, while the high angle grain boundaries (HAGBs $\theta>15^{\circ}$ ) are labelled in black. Grain size calculation was done using TSL software, employing the intercept method. The grain size distribution curves were drawn using TSL software, from which the minimum grain size of the material at respective deformation temperature and strain were calculated. The larger grains seen in the microstructure were manually separated out and drawn using GIMP ${ }^{\circledR}$ software, and then quantified using the image analysis software, MIP $^{\circledR}$ (Nahamin Pardazan Asia Co., Iran). These grains are associated with the maximum grain size of the material at different deformation conditions.

\section{Results}

\subsection{Stress - Strain Curves}

The flow stress-strain curves obtained after deformation at $1200{ }^{\circ} \mathrm{C}$ and $1150{ }^{\circ} \mathrm{C}$, and strain rates of $0.25 \mathrm{~s}^{-1}, 1 \mathrm{~s}^{-1}$ and $2 \mathrm{~s}^{-1}$ are displayed in Fig. 4. Note that these temperatures are approximately $450{ }^{\circ} \mathrm{C}$ and $400{ }^{\circ} \mathrm{C}$, respectively, above its paraequilibrium ( $\left.\mathrm{Ae}_{3}\right)$ temperature. The results show that the stress levels increase with an increase in strain rate; however, it decreases with an increase in temperature. The steady state flow was attained at a strain of about 0.5. At a strain rate of $0.25 \mathrm{~s}^{-1}$, curves show an initial increase in stress, which is then accompanied by a gradual drop to a steady state value. With the increase in strain rate, true stress-true strain curves for both deformation temperatures show typical softening behavior. A stress drop of approximately $15 \%$ can be seen at testing conditions, employing the lowest strain rate and highest temperature, indicating the occurrence of dynamic softening phenomena. However, it is well established that 
during hot deformation of medium-carbon low-alloy steels, in addition to DRX, the DT of austenite to ferrite, whose kinetics is much faster than DRX, may also occur [23]. The double differentiation technique, proposed by Poliak and Jonas [24, 25], was used to evaluate the critical strains and stresses for the initiation of both DT and DRX. A dedicated script was developed in MATLAB $^{\circledR}$ for analyzing the flow curves using this method.

In order to determine the critical stresses for the initiation of DT and DRX, the strain hardening rate, $\theta$, was calculated by the following expression:

$$
\theta=\left(\frac{\delta \sigma}{\delta \varepsilon}\right)_{\dot{\varepsilon}}
$$

The minima were then calculated by taking the derivative of $\theta,[26,27]$ :

$$
\left(\frac{\delta}{\delta \sigma}\right)\left(\frac{\delta \theta}{\delta \sigma}\right)=0
$$

The two minima shown in Fig. 5 represent the critical stresses at which DT and DRX were initiated during deformation. The critical strains corresponding to these stresses were then calculated using the initial flow curves reported in Fig. 4. The plots in Fig. 6 show variations of DT and DRX critical strains versus temperature and strain rate. This finding is in agreement with those reported by other researchers, who also showed that the critical strain for DT is less than that of DRX [2729]. Observations from the calculation of equations (1) and (2) of the present alloy show that the critical strain for DT is less than the one needed for DRX. In the present study the critical strains were 0.08 for DT and 0.13 for DRX at a deformation temperature of $1200{ }^{\circ} \mathrm{C}$ and a strain rate of $0.25 \mathrm{~s}^{-1}$. From Fig. 6, it can be depicted that the critical strain for the initiation of DRX is higher than that for DT. It decreases with an increase in temperature and an increase in strain rate.

However, it must be noted that, the double differentiation technique is purely mathematical, and therefore microstructure analysis is needed to show the physical significance of critical strain on the evolution of the microstructure during hot deformation. 


\subsection{Microstructure Analysis}

Fig. 7 shows the grain boundary map of the specimen deformed at $1200{ }^{\circ} \mathrm{C}$ and at strain rates of $0.25 \mathrm{~s}^{-1}$ (Fig. 7 (a)), $1 \mathrm{~s}^{-1}$ (Fig. 7 (b)) and $2 \mathrm{~s}^{-1}$ (Fig. 7 (c)). The microstructure shows prior austenite grain boundaries (shown in black), confirming the large grain size of prior austenite. At a higher strain rate $\left(2 \mathrm{~s}^{-1}\right)$, a structure with a Widmanstätten type morphology is observed, which originates from the prior austenite grain boundaries and the interior of the grains. Note that ferrite plates were formed instead of martensitic structures due to the applied stress, which induces the displacive transformation of austenite to Widmanstätten ferrite. The width of these plates is affected by temperature due to the diffusion of alloying elements such as carbon. This observation is consistent with the results of numerous researchers $[8,9,14]$. When the strain rate decreases to $1 \mathrm{~s}^{-1}$, the microstructure becomes slightly coarser and the morphology appears to be mixed with lath and a few quasi-polygonal grains (see encircled area). At the lowest strain rate, a polygonal grain type morphology originating from the grain boundaries and from the interior of the grains was observed. Few polygonal grains show serrated and bulged grain boundaries, indicating the nucleation of new grains from these bulged areas of the grain boundaries (shown with red arrows). Comparative study of the features of the microstructure revealed that the Widmanstätten ferrite plates were finer at higher strain rates (e.g., Figs. 7 (b) \& (c)). It is believed that this may be due to the disintegration of the deformation-induced Widmanstätten ferrite plates under high strain rate conditions, resulting in a finer structure (encircled in Fig. 7 (c)).

Fig. 8 shows the grain boundary map of the specimen deformed at $1150{ }^{\circ} \mathrm{C}$ and at strain rates of $0.25 \mathrm{~s}^{-1}$ (Fig. 8 (a)), $1 \mathrm{~s}^{-1}$ (Fig. 8 (b)) and $2 \mathrm{~s}^{-1}$ (Fig. 8 (c)). At a strain rate of $2 \mathrm{~s}^{-1}$ (Fig. 8 (c)), the microstructure is slightly finer than the one observed in Fig. 7 (c), i.e., Widmanstätten plates, nucleating from the prior austenite grain boundaries (highlighted with yellow arrow). At the intermediate strain rate, the microstructure is composed of lath and quasipolygonal type ferrite. A comparison between the ferrite morphologies at deformation temperatures of $1150{ }^{\circ} \mathrm{C}$ and $1200{ }^{\circ} \mathrm{C}$ and strain rate of $0.25 \mathrm{~s}^{-1}$ showed that, while in both cases the dominant component of the microstructure is made of quasi-polygonal type ferrite, Widmanstätten plates are clearly visible in the sample deformed at $1150{ }^{\circ} \mathrm{C}$ and none in the one deformed at $1200{ }^{\circ} \mathrm{C}$ (Fig. 7 (a)). 
Hot deformed microstructures at $1200{ }^{\circ} \mathrm{C}$ and $1150{ }^{\circ} \mathrm{C}$ for all the strain rates revealed a microstructure consisting of colonies of bainite laths with different morphologies, and polygonal grains in different orientations with respect to the loading directions. A detailed microstructure analysis using EBSD grain boundary maps showed that at a strain rate of $0.25 \mathrm{~s}^{-1}$, for the material deformed at $1200{ }^{\circ} \mathrm{C}$, the entire microstructure was free from low angle grain boundaries (LAGBs), which could be an indication that the microstructure is fully recovered, although recrystallization and phase transformation may also contribute to a decrease in LAGBs. In contrast, LAGBs were still present in the sample deformed at $1150{ }^{\circ} \mathrm{C}$ and at a similar strain rate, suggesting that this temperature was insufficient to fully recover the microstructure. The polygonal grains displayed in Figs. 7 (a) and 8 (a) $\left(0.25 \mathrm{~s}^{-1}\right)$ with few LAGBs or no LAGBs are strain-free grains. Their presence is probably due to the fact that a low strain rate and high temperature provide a sufficient driving force for recovery [30]. Figs. 7 (c) and 8 (c) show that significantly higher fractions of LAGBs are present in the grain boundary maps of the material deformed at higher strain rates (i.e., at $2 \mathrm{~s}^{-1}$ ), which indicates that the internal structure of these lathes is highly deformed.

\section{Discussion}

\subsection{Flow Curves Analysis}

\subsubsection{Effect of Strain Rate}

An analysis of Fig. 6 shows that with the increase in strain rate from $0.25 \mathrm{~s}^{-1}$ to $2 \mathrm{~s}^{-1}$, critical strains increase by about $40 \%$ (for $\mathrm{T}=1200{ }^{\circ} \mathrm{C}$ ). Here, it is important to note that although the critical strain for the initiation of DT is higher at higher strain rates $\left(\varepsilon_{\mathrm{c}}=0.103\right.$ at $2 \mathrm{~s}^{-1}$ as compared to $\varepsilon_{\mathrm{c}}=0.073$ at $0.25 \mathrm{~s}^{-1}$ for $\mathrm{T}=1200^{\circ} \mathrm{C}$ ), the time to reach this critical strain is much shorter since the strain rate is higher $\left(0.05 \mathrm{~s}\right.$ for $2 \mathrm{~s}^{-1}$ and $0.4 \mathrm{~s}$ for $\left.0.25 \mathrm{~s}^{-1}\right)$. The above results reveal a clear influence of strain rate on the initiation of DT. These observations are in contrast with some previous reports, which indicate that the strain rate does not affect the DT critical strains and/or the initiation of dynamic transformation [26, 31]. The influence of strain rate on the formation of ferrite from deformed austenite can be justified in terms of the available time for dynamic transformation at lower strain rates and the diffusion of alloying elements. This will be discussed in detail in the next sections. 


\subsubsection{Effect of Temperature}

The influence of temperature on the initiation of DT is illustrated in Fig. 6. The temperature effect on the critical strains associated with the initiation of DT is well established in the literature $[9,12,14-16,23,32]$. It has been reported that the critical strain increases with the temperature beyond $\mathrm{Ae}_{3}$ to a maximum value, but then decreases until it reaches the delta ferrite formation temperature [14]. From a thermodynamic perspective, this could be related to the free energy for transformation. The evaluation of the energy barrier, defined here as the difference between the $\Delta G$ of ferrite and austenite, was calculated for the present steel using the Factsage thermodynamic software, employing the FSStel Database ${ }^{\circledR}$ [21]. As shown in Fig. 9, $\Delta G$ for DT starts to decrease from $1090{ }^{\circ} \mathrm{C}$. The lower energy barrier value also results in less work hardening required for the transformation softening to take place in austenite. As indicated in Fig. 6, the critical strains at $1200{ }^{\circ} \mathrm{C}$ are lower than those at $1150{ }^{\circ} \mathrm{C}$ for all strain rates. Here, $1200{ }^{\circ} \mathrm{C}$ is closer to the delta

ferrite formation temperature of this alloy, which is approximately $1480^{\circ} \mathrm{C}$, as calculated with the Thermocalc $^{\circledR}$ Software, using the TCFE7 database [33]. As the deformation temperature approaches the delta ferrite formation temperature, the Gibbs free energy of the deformed austenite gets very close to that of the delta ferrite, thereby easing the initiation of DT. However, it should be noted that the critical strain measured in this study is higher than that reported in the literature (0.11, compared to 0.08 for $\mathrm{T}=1200{ }^{\circ} \mathrm{C}$ and $\dot{\varepsilon}=1 \mathrm{~s}^{-1}$ ) [14,32]. The difference in the critical strain value is probably due to the higher carbon level in the present steel $(0.34 \%)$ as compared to the carbon level reported for low carbon steel (0.06 \%) [32]. Indeed, as reported by Ghosh et al. [11], the resistance to DT increases with an increase in the carbon content of steel for the same testing temperature because carbon is an austenite stabilizer. Note that the addition of austenite stabilizers is known to decrease the $\mathrm{Ae}_{3}$ temperature. Considering the fact that lower $\mathrm{Ae}_{3}$ values delay the initiation of dynamic transformation, higher values of DT critical strain are therefore expected in the present steel.

\subsection{Analysis of Grain Boundary Maps}

The grain size of the starting material (as-cast and homogenized) was about $\sim 400 \mu \mathrm{m}$ (Fig. 3). This value is very large in comparison with all other previous studies: $13 \mu \mathrm{m}$ [34], $15 \mu \mathrm{m}$ [13], $20 \mu \mathrm{m}$ [35] and $65 \mu \mathrm{m}$ [7]. Based on the obtained results, the microstructure after deformation is expected to be composed of DRXed austenite and DTed ferrite. After quenching, the 
microstructure is expected to be composed of martensite and DTed ferrite. However, it is difficult to differentiate between martensite and dynamically transformed ferrite by using XRD, optical, or secondary electron imaging [7]. In the present study, the EBSD technique, and specifically the Kernal Average Misorientation (KAM) [36] method, was used to obtain internal misorientation between grains, and to therefore distinguish the BCT martensite from the BCC ferrite.

Fig. 10 shows schematic representation of the KAM approach [36, 37]. The average misorientation of a given point with all points (Fig. 10 (a)) in the kernel or at the perimeter of the kernel is calculated with the provision that misorientations exceeding a given threshold value are excluded. In other words, by using KAM, grain size is defined based on a certain angle of grain boundaries and number of step sizes. In the present work, up to the third nearest neighbor (step size) was considered for calculating KAM values, and a threshold angle of $5^{\circ}$ was used. To study the recovery behavior, only the area fractions with less than $2^{\circ}$ misorientation (i.e., $\mathrm{KAM} \leq 2^{\circ}$; green and blue regions) were considered.

Using the above criteria, the dynamic recovery process of the two phases was studied, and a differentiation was made between ferrite and austenite. It is known that ferrite has a higher stacking fault energy (SFE) [38] than austenite, which would make dynamic recovery easier when it is further deformed to a higher strain $(\varepsilon=0.8)$. This leads to lower density of LAGBs within ferrite grains, which in turn leads to lower misorientation within the grain, as confirmed in the KAM map shown in Fig. 11. On the other hand, martensite laths (from prior austenite) which are formed due to shape deformation (displacive transformation) generate a higher amount of LAGBs, resulting in higher misorientations within the laths.

Fig. 11 (a) shows the KAM map for the specimen deformed at $1200{ }^{\circ} \mathrm{C}$ and at a strain rate of $0.25 \mathrm{~s}^{-1}$. Quasi-polygonal ferrite grains, as observed on the Grain Boundary map (G.B. map) of Fig. 7 (a), have $<2^{\circ}$ misorientation (Fig. 11 (a)), thus confirming that they are recovered ferrite grains, whereas at $1200{ }^{\circ} \mathrm{C}$ and at a strain rate of $2 \mathrm{~s}^{-1}$, as shown in Fig. 11 (c), the majority of the grains have a KAM between $3^{\circ}-5^{\circ}$, signifying the presence of martensite due to untransformed austenite.

Fig. 12 shows the approximate fraction of ferrite $\left(\right.$ KAM values $\left.<2^{\circ}\right)$ as a function of the Zener Hollomon parameter (Z parameter, equation (3) [22, 39]). 


$$
Z=\dot{\varepsilon} \exp \left(\frac{Q}{R T}\right)
$$

The area fraction of KAM with misorientations of less than $2^{\circ}$ represent the ferrite volume fraction. The ferrite volume fraction decreases as the strain rate increases, while it is higher at higher temperatures. For instance, at the strain rates of $0.25 \mathrm{~s}^{-1}$ and $2 \mathrm{~s}^{-1}$, the ferrite volume fraction was around $77 \%$ and $38 \%$, respectively. Similarly, at a constant strain rate of $0.25 \mathrm{~s}^{-1}$, the ferrite volume fraction was respectively $77 \%$ and $72 \%$ at $1200{ }^{\circ} \mathrm{C}$ and $1150{ }^{\circ} \mathrm{C}$. The above findings are consistent with those reported by N. Park et al. $[8,40]$ who observed that with the decrease in the strain rate, the ferrite volume fraction increased in a $6 \mathrm{Ni}-0.1 \mathrm{C}$ steel. Considering that the growth of a dynamically transformed ferrite plate depends on the diffusion of alloying elements during deformation, and particularly that of carbon, the influence of the strain rate and temperature on the occurrence of DT can, therefore, be correlated to the diffusion of carbon during deformation. This will be discussed in the following section.

\subsection{Influence of Strain Rate on Carbon Diffusion}

Several authors have reported on increase in carbon diffusion with temperature $[9,14,23,32]$. Specifically, in DT studies on medium-carbon low-alloy steels, it has been reported that carbon diffusivity increases by 8.6 times when the temperature increases from $900{ }^{\circ} \mathrm{C}$ to $1350{ }^{\circ} \mathrm{C}$ [1-7]. However, this increase is not solely associated with temperature change, but is also related to the applied stress. The influence of strain rate on carbon diffusivity can be quantified by plotting the diffusivity of carbon vs. strain. Indeed, as the stress values for each interval of strain vary with the strain rate, the effect of strain rate on carbon diffusion can be calculated by incorporating the respective stress values at a particular strain with respect to the individual strain rates, in the diffusion equations.

Lattice and pipe diffusion of carbon are the two important mechanisms by which solutes transfer to or away from stacking faults [41]. Lattice diffusion occurs when substitutional or interstitial atoms move to another lattice position, e.g., Fe atoms in $\mathrm{Fe}$ alloys, whereas pipe diffusion occurs when the atoms move via edge dislocations e.g. interstitial atoms like carbon in Fe alloys $[42,43]$. It is known that the interstitial atoms and solutes diffuse much faster through pipe diffusion than lattice diffusion due to higher activation energies required for the latter [44]. 
Therefore, pipe diffusion plays the most important role, and is probably the dominant mechanism in the diffusion of solutes $[45,46]$

The effective diffusion is given by $D_{\text {eff }}$ as;

$$
D_{\text {eff }}=D_{L} f_{L}+D_{P} f_{P}
$$

where $D_{L}$ and $D_{P}$ are respectively the lattice and pipe diffusion coefficients, $f_{L}$ and $f_{P}$ are fractions of atoms participating in the lattice and pipe diffusion processes, and $f_{L}$ is given as unity and $f_{P}=$ $7.03125\left(\frac{\sigma}{\mu}\right)^{2}[47]$.

The general expression for calculating $D_{L}$ and $D_{P}$ are presented in Appendix 1, equations A5 \& A6. In these equations, $b$ represents the Burgers vector $(m)$ and $\mu$ represents shear modulus (MPa). The equations for obtaining the values of $b$ (eq. A1) and $\mu$ (eq. A3) under applied stress were proposed by Ashby and Frost [48, 49]. It is shown from the equations that when there is no stress, there is no pipe diffusion.

Table 2 shows all the required variables for the calculation of $D_{L}$ and $D_{P}$ of carbon.

The diffusivity due to $D_{L}, D_{P}$ and the corresponding effective diffusion $\left(D_{\text {eff }}\right)$ are shown in Fig. 13 for a deformation temperature of $1200^{\circ} \mathrm{C}$ and a strain rate of $0.25 \mathrm{~s}^{-1}$. It can be seen that $D_{L}$ is constant while $D_{P}$ changes with change in stress value at constant temperature. Specifically, $D_{P}$ was found to be 6.8 times faster than $D_{L}$, indicating the significance of $D_{P}$. Since lattice diffusion is constant for a particular temperature and independent of the stress level, only the variations of $D_{P}$ will be considered for other temperatures. Diffusivity due to pipe diffusion (eq. A6) for a deformation temperature of (a) $1200^{\circ} \mathrm{C}$ and (b) $1150{ }^{\circ} \mathrm{C}$, respectively, at various strain levels, is shown in Fig 14. It can be seen that at a constant temperature, the diffusivity of carbon increases with an increase in the strain rate. The equations used to calculate $D_{P}$ have stress as a variable, thereby indicating that any change in the stress value should significantly change the diffusivity values at a constant temperature. Therefore, the application of the compressive stress during deformation accelerates the pipe diffusion process (i.e., $D_{P}$ increases). The obtained results are in good agreement with those reported by Tingdong [50], who for a series of low carbon steels, 
observed that the diffusion coefficient of impurity-vacancy complex was three times larger under applied stress than under stress-free conditions.

Fig. 15 shows the distances over which carbon atoms can diffuse under lattice and pipe diffusion at $1200{ }^{\circ} \mathrm{C}$ and at a strain rate of $0.25 \mathrm{~s}^{-1}$. It can be seen that the lattice diffusion distance varies from $0.48 \mu \mathrm{m}$ (at $\varepsilon=0.05)$ to $1.90 \mu \mathrm{m}(\varepsilon=0.8)$ at $1200{ }^{\circ} \mathrm{C}$, whereas the pipe diffusion distance varies from $1.15 \mu \mathrm{m}$ (at $\varepsilon=0.05)$ to $4.80 \mu \mathrm{m}(\varepsilon=0.8)$. Specifically, at a strain of 0.8 , the distance traveled by pipe diffusion is $\sim 2.4$ times higher than that by lattice diffusion. Figs. 16 ((a) $\&$ (b)) show the variation of diffusion distance, $\mathrm{x}(\mu \mathrm{m})$ due to pipe diffusion vs. strain at a deformation temperature of (a) $1200{ }^{\circ} \mathrm{C}$ and (b) $1150{ }^{\circ} \mathrm{C}$. The results indicate that the diffusion distance traveled by carbon atoms decreases when the strain rate increases. As shown in Fig. 14, as the stress increases, the diffusivity increases, but with the increase in strain rate, the trend is opposite. Furthermore, it appears that the time available for the diffusion is less at high strain rates than at slower strain rates. For example, at a strain of 0.8 , this time is $0.4 \mathrm{~s}$ at $2 \mathrm{~s}^{-1}$ while it is $3.2 \mathrm{~s}$ at $0.25 \mathrm{~s}^{-1}$. This indicates that the governing mechanism controlling the diffusion of carbon from ferrite, after transformation, is mainly dislocation pipe diffusion. This finding is in agreement with those reported by other researchers who identified dislocation pipe diffusion as the governing mechanisms during hot deformation of other steels $[46,51,52]$. Finally, Figs. 17 ((a) \& (b)) show the variation of diffusion distance, $\mathrm{x}(\mu \mathrm{m})$, due to pipe diffusion vs. deformation time, $\mathrm{t}(\mathrm{s})$ for a strain rate of $0.25 \mathrm{~s}^{-1}$ (a) and $2 \mathrm{~s}^{-1}$ (b). An analysis of the graph shows that with an increase in both temperature and deformation time, the diffusion distance of carbon atoms increases. This finding could be correlated with the higher volume fraction of ferrite obtained after deformation at a strain rate of $0.25 \mathrm{~s}^{-1}$, where the deformation time is 8 times higher, than when the deformation was applied with a strain rate of $2 \mathrm{~s}^{-1}$.

\subsubsection{Evaluation of the DT Ferrite Grain Size}

The nucleation and growth of DT ferrite depends on several factors, such as temperature, stress level, alloying elements, holding time, etc. These have been studied in detail by various researchers [9]. Due to the complexity of separating the individual effects of these factors, the present results account for the influence of carbon diffusion in the nucleation and growth of ferrite. The evolution of ferrite grain size is certainly related to the diffusion distance of 
carbon $[9,53]$. In this study, a new mechanism is proposed in order to relate the grain size to the diffusion distance. To that end, the following assumptions are made:

1. The grain size at the critical strain for DT (i.e., at $\varepsilon=0.1$ ) is assumed as the smallest average grain size in the GB map (Figs. $7 \& 8$ ).

2. The largest average grain size for the strain of $\varepsilon=0.8$ is the grain size of the large grains observed in the GB map (Figs. $7 \& 8$ ).

The calculation followed the procedure described in the Materials and Methods section.

As per the diffusion calculation presented in Fig. 16, the critical strain for the occurrence of DT corresponds to the minimum distance traveled by the carbon atoms, whereas the highest strain corresponds to the maximum distance travelled by the carbon atoms.

Fig. 18 shows the plot of the smallest and largest average grain sizes with respect to the diffusion distance of carbon for strain rates of (a) $0.25 \mathrm{~s}^{-1}$ and (b) $2 \mathrm{~s}^{-1}$. It can be seen that with the increase in the strain rate, the diffusion distance of carbon decreases with respect to the smallest or largest average grain size.

The relation between the grain size evolution and the diffusion distance of carbon for strain rates of (a) $0.25 \mathrm{~s}^{-1}$ and (b) $2 \mathrm{~s}^{-1}$ is shown schematically in Fig. 19.

During deformation at the lowest strain rate, i.e., $0.25 \mathrm{~s}^{-1}$ (Fig. 19 (a)), at the stage I, nucleation of DT Widmanstätten ferrite plates from the prior austenite grain boundaries (PAGBs) starts. The nucleated plates are "self-accommodating plates", which form due to the shear and dilatation of austenite [15]. At this stage, the diffusion distance (1.85 $\mu \mathrm{m}$, Fig. 18) is greater than the smallest average grain size $(1.11 \mu \mathrm{m})$, and therefore, the carbon atoms can diffuse out of the Widmanstätten ferrite plates from both the width and the leading edges. With increasing deformation, the nucleated DT Widmanstätten ferrite continues to expand both from the edges and the width, while at the same time, more nucleation of DT Widmanstätten ferrite takes place from the PAGBs, as well as in the interior of the grains. This has been related to the occurrence of variable amounts of stored energy within different parts of large grains, due to the activation of different slip systems $[31,54]$. The width and the length of the plates keep on increasing from all directions until they start to coalesce with other plates to form grains. Specifically, in stage II, the 
diffusion distance of carbon $(5.26 \mu \mathrm{m})$ is significantly greater than that of the largest average grain size $(4.26 \mu \mathrm{m})$, therefore the plates coalesce into grains, and a lath structure is produced. The features of the lath type structure are clearly visible in Fig. 7 (a), and partially in Fig. 8 (a).

During deformation at the highest strain rate, i.e., $2 \mathrm{~s}^{-1}$ (Fig. 19 (b)), at stage I, nucleation of DT Widmanstätten ferrite grains from the PAGBs initiates from the critical strain of $\varepsilon=0.1$. At this stage, the diffusion distance $(0.58 \mu \mathrm{m})$ is smaller than the smallest average grain size $(0.77$ $\mu \mathrm{m})$, and therefore, carbon atoms cannot diffuse out of the ferrite from the width direction, whereas they can diffuse out from the leading edges. Moreover, the growth rate of the Widmanstätten plate depends on the diffusivity of carbon atoms [17]. As a result, the plates tend to increase in the leading edge in the direction of the applied stress. Further straining causes more nucleation of the DT Widmanstätten ferrite due to the activation of more slip systems. Here, the plates are not seen growing in the width direction, and therefore, no grains are formed, as in case of low strain rate $\left(0.25 \mathrm{~s}^{-1}\right)$. With the increase in strain, the plates start to disintegrate as they further increase lengthwise [14]. Clear evidence of this behavior is shown in Figs. 7 (c) and 8 (c), where long and thin Widmanstätten ferrite plates and their gradual disintegration are clearly visible.

In summary, the formation mechanism of ferrite grains is a two-step process, where ferrite nucleates first as Widmanstätten type laths, and in the second stage the laths coalesce due to the least misorientation between two adjacent ferrite plates and convert into granular morphology [14, $23,31,32]$. However, the coalescence process cannot be accomplished during high strain rate deformations due to the higher misorientation difference between two adjacent laths produced by intense straining, whereas at higher temperature and lower strain rates, the microstructure is in recovery state.

\section{Conclusions}

The possible occurrence of dynamic transformation of austenite to ferrite in the as-cast structure of a large size ingot of medium-carbon low-alloy steel and the influence of strain rate on the occurrence of DT were investigated. Based on the analysis of the flow curves by means of double differentiation method and microstructural analysis using electron microscopy, it appears that DT takes place even in very large grain sized materials. Moreover, more ferrite was observed at around $1200{ }^{\circ} \mathrm{C}$, which is close to the formation temperature of delta ferrite. 
By employing the KAM technique, it was determined that deformation at high strain rates resulted in a reduction in the fraction of dynamically transformed ferrite. It was also found that deformation at high strain rates produced DT ferrite with Widmanstätten morphology, while at a lower strain rate, quasi-polygonal morphology was found.

Analysis of carbon diffusion showed higher diffusivity and shorter diffusion distances at higher strain rates. On this basis, correlations between the ferrite fraction, the diffusion distance of carbon atoms, and grain size was established and the influence of changes in stress level due to strain rate and temperature on DT ferrite and its growth were determined.

A better understanding of the mechanisms and kinetics of DT ferrite formation in as-cast materials contribute to a better prediction of the final microstructure at the end of the ingot breakdown process of large size ingots. By accounting the occurrence of DT (in combination with DRX, DRV and MDRX) during high temperature deformation processing, more precise thermomechanical schedules and cooling models can be developed.

\section{Acknowledgements}

The authors are very grateful to Finkl Steel for providing the specimens for the present research. They would also like to thank the National Science Engineering Research Council (NSERC) Canada for their support in the framework of a Collaborative Research and Development project (CRD). One of the authors (Kanwal Chadha) would like to acknowledge the support of ÉTS, Canada, for financial support in visiting the Indian Institute of Technology, Hyderabad, India, to carry out the EBSD characterization. We would also like to thank Dr. Pinaki Prasad Bhattacharjee for his valuable input in the analysis of EBSD images and Mr. Rajasekhar Reddy Seelam for assisting in the post-processing of EBSD data.

\section{Appendix I}

In order to estimate the diffusion distance of carbon using effective, pipe and lattice diffusion parameters (eq. 4), the coefficients used are determined as follows:

$$
b\left(\sigma_{s}\right)=b_{0} \exp \left(-\frac{\sigma_{s}}{3 K}\right)
$$




$$
\begin{aligned}
& K\left(T, \sigma_{s}\right)=K_{0}\left[1+\frac{T_{M} d K}{K_{0} d T}\left(\frac{T-300}{T_{M}}\right)\right]+\frac{\sigma_{s} d K}{K_{0} d \sigma_{s}} \\
& \mu\left(T, \sigma_{s}\right)=\mu_{0}\left[1+\frac{T_{M} d K}{\mu_{0} d T}\left(\frac{T-300}{T_{M}}\right)\right]+\frac{\sigma_{s} d \mu}{\mu_{0} d \sigma_{s}} \\
& Q_{p}=Q_{p 0}+\sigma_{s} V_{p}^{*} \\
& D_{L}=D_{0 L} \exp \left(-\frac{Q_{L}}{R T}\right) \\
& D_{P e f f}=\frac{10}{b^{2}}\left(\frac{\sigma_{s}}{\mu}\right)^{2} a_{p} D_{p} \\
& a_{p} D_{p}=a_{p} D_{0 p} \exp \left(-\frac{Q_{p}}{R T}\right)
\end{aligned}
$$

\section{References:}

1. Reed-Hill, R.E., R. Abbaschian, and R. Abbaschian, Physical metallurgy principles. 1973.

2. Sakai, T., M. Akben, and J. Jonas, Dynamic recrystallization during the transient deformation of a vanadium microalloyed steel. Acta Metallurgica, 1983. 31(4): p. 631-641.

3. Devadas, C., I. Samarasekera, and E. Hawbolt, The thermal and metallurgical state of steel strip during hot rolling: Part III. Microstructural evolution. Metallurgical Transactions A, 1991. 22(2): p. 335-349.

4. Laasraoui, A. and J. Jonas, Prediction of steel flow stresses at high temperatures and strain rates. Metallurgical transactions A, 1991. 22(7): p. 1545-1558. 
5. Matsumura, Y. and H. Yada, Evolution of Ultrafine-grained Ferrite in Hot Successive Deformation. Transactions of the Iron and Steel Institute of Japan, 1987. 27(6): p. 492-498.

6. Yada, H., C.M. Li, and H. Yamagata, Dynamic $\gamma \rightarrow \alpha$ Transformation during Hot Deformation in Iron-Nickel-Carbon Alloys. ISIJ International, 2000. 40(2): p. 200-206.

7. Sun, L., et al., Effect of strain path on dynamic strain-induced transformation in a microalloyed steel. Acta Materialia, 2014. 66: p. 132-149.

8. Park, N., et al., Flow stress analysis for determining the critical condition of dynamic ferrite transformation in 6Ni-0.1C steel. Acta Materialia, 2013. 61(1): p. 163-173.

9. Ghosh, C., C. Aranas Jr, and J.J. Jonas, Dynamic transformation of deformed austenite at temperatures above the Aes. Progress in Materials Science, 2016. 82: p. 151-233.

10. Ghosh, C., et al., The dynamic transformation of deformed austenite at temperatures above the Aes. Acta Materialia, 2013. 61(7): p. 2348-2362.

11. Ghosh, C., V.V. Basabe, and J.J. Jonas, Thermodynamics of dynamic transformation of hot deformed austenite in four steels of increasing carbon contents. Materials Science and Engineering: A, 2014. 591: p. 173-182.

12. Aranas Jr, C. and J.J. Jonas, Effect of Mn and Si on the dynamic transformation of austenite above the $\mathrm{Ae}_{3}$ temperature. Acta Materialia, 2015. 82: p. 1-10.

13. Park, N., et al., Effect of austenite grain size on kinetics of dynamic ferrite transformation in low carbon steel. Scripta Materialia, 2013. 68(8): p. 611-614.

14. Grewal, R., et al., Formation of Widmanstätten ferrite at very high temperatures in the austenite phase field. Acta Materialia, 2016. 109: p. 23-31.

15. Jonas, J.J. and C. Ghosh, Role of mechanical activation in the dynamic transformation of austenite. Acta Materialia, 2013. 61(16): p. 6125-6131.

16. Aranas, J.C., T. Wang, and J.J. Jonas, Effect of Interpass Time on the Dynamic Transformation of a Plain $\mathrm{C}-\mathrm{Mn}$ and a Nb Microalloyed Steel. ISIJ International, 2015. 55(3): p. 647-654.

17. Ali, A. and H. Bhadeshia, Nucleation of Widmanstätten ferrite. Materials Science and Technology, 1990. 6(8): p. 781-784.

18. Zhao, L., et al., Combination of dynamic transformation and dynamic recrystallization for realizing ultrafine-grained steels with superior mechanical properties. Scientific Reports, 2016. 6: p. 39127.

19. Ohmori, A., et al., Effect of deformation temperature and strain rate on evolution of ultrafine grained structure through single-pass large-strain warm deformation in a low carbon steel. Materials transactions, 2004. 45(7): p. 2224-2231.

20. Tsuji, N., Y. Matsubara, and Y. Saito, Dynamic recrystallization of ferrite in interstitial free steel. Scripta materialia, 1997. 37(4).

21. Bale, C.W., et al., FactSage thermochemical software and databases - recent developments. Calphad, 2009. 33(2): p. 295-311.

22. Chadha, K., et al., Deformation and Recrystallization Behavior of the Cast Structure in Large Size, High Strength Steel Ingots: Experimentation and Modeling. Metallurgical and Materials Transactions A, 2017. 48(9): p. 4297-4313.

23. Aranas Jr, C., et al., Flow Softening-based Formation of Widmanstätten Ferrite in a 0.06\% C Steel Deformed Above the Aes. ISIJ International, 2015. 55(1): p. 300-307.

24. Poliak, E.I. and J.J. Jonas, Critical strain for dynamic recrystallization in variable strain rate hot deformation. ISIJ International, 2003. 43(5): p. 692-700. 
25. Poliak, E.I. and J.J. Jonas, A one-parameter approach to determining the critical conditions for the initiation of dynamic recrystallization. Acta Materialia, 1996. 44(1): p. 127-136.

26. Jonas, J.J., et al., The Critical Strain for Dynamic Transformation in Hot Deformed Austenite. ISIJ International, 2013. 53(1): p. 145-151.

27. Ghosh, C., V.V. Basabe, and J.J. Jonas, Determination of the Critical Strains for the Initiation of Dynamic Transformation and Dynamic Recrystallization in Four Steels of Increasing Carbon Contents. steel research international, 2013. 84(5): p. 490-494.

28. Sun, X., et al., Microstructural Evolution and Kinetics for Post-dynamic Transformation in a Plain Low Carbon Steel. ISIJ International, 2008. 48(7): p. 994-1000.

29. Choi, J.K., et al., Formation of ultrafine ferrite by strain-induced dynamic transformation in plain low carbon steel. ISIJ International, 2003. 43(5): p. 746-754.

30. Rollett, A., et al., Recrystallization and related annealing phenomena. 2004: Elsevier.

31. Basabe, V.V., J.J. Jonas, and C. Ghosh, Formation of Strain-induced Ferrite in Low Carbon Steels at Temperatures Above the Aes. ISIJ International, 2013. 53(12): p. 22332241.

32. Jr., C.A., et al. Formation of Widmanstätten Ferrite in a C-Mn Steel at Temperatures High in the Austenite Phase Field. in Proceedings of the International Conference on Solid-Solid Phase Transformations in Inorganic Materials (PTM 2015). 2015. Whistler, BC.

33. Andersson, J.O., et al., Thermo-Calc \& DICTRA, computational tools for materials science. Calphad, 2002. 26(2): p. 273-312.

34. Eghbali, B., Study on the ferrite grain refinement during intercritical deformation of a microalloyed steel. Materials Science and Engineering: A, 2010. 527(15): p. 3407-3410.

35. Cai, M.H., H. Ding, and Y.K. Lee, Dynamic Strain-Induced Ferrite Transformation during Hot Compression of Low Carbon Si-Mn Steels. MATERIALS TRANSACTIONS, 2011. 52(9): p. 1722-1727.

36. Schwartz, A.J., et al., Electron Backscatter Diffraction in Materials Science: Springer Science 2010

37. Bhattacharjee, P.P., et al., Evolution of Microstructure and Texture During Warm Rolling of a Duplex Steel. Metallurgical and Materials Transactions A, 2014. 45(4): p. 2180-2191.

38. Reick, W., M. Pohl, and A.F. Padilha, Recrystallization and Related Phenomena. Recrystallization-Transformation Combined Reactions during Annealing of a Cold Rolled Ferritic-Austenitic Duplex Stainless Steel. ISIJ International, 1998. 38(6): p. 567-571.

39. Chadha, K., D. Shahriari, and M. Jahazi, Constitutive Modelling of Ingot Breakdown Process of Low Alloy Steels. La Metallurgia Italiana - International Journal of the Italian Association for Metallurgy, 2016. 4: p. 5-12.

40. Park, N., et al., Occurrence of dynamic ferrite transformation in low-carbon steel above $\mathrm{Ae}_{3}$. Scripta Materialia, 2013. 68(7): p. 538-541.

41. Viswanathan, G.B., et al., Segregation at stacking faults within the $\gamma^{\prime}$ phase of two Ni-base superalloys following intermediate temperature creep. Scripta Materialia, 2015. 94(Supplement C): p. 5-8.

42. Love, G.R., Dislocation pipe diffusion. Acta Metallurgica, 1964. 12(6): p. 731-737.

43. Levine, H.S. and C.J. MacCallum, Grain Boundary and Lattice Diffusion in Polycrystalline Bodies. Journal of Applied Physics, 1960. 31(3): p. 595-599.

44. Porter, D.A., K.E. Easterling, and M. Sherif, Phase Transformations in Metals and Alloys. 2009: CRC press. 
45. Robinson, S.L. and O.D. Sherby, Mechanical behavior of polycrystalline tungsten at elevated temperature. Acta Metallurgica, 1969. 17(2): p. 109-125.

46. Sauvage, X. and Y. Ivanisenko, The role of carbon segregation on nanocrystallisation of pearlitic steels processed by severe plastic deformation. Journal of Materials Science, 2007. 42(5): p. 1615-1621.

47. Samantaray, D., et al., Strain dependent rate equation to predict elevated temperature flow behavior of modified 9Cr-1Mo (P91) steel. Materials Science and Engineering: A, 2011. 528(3): p. 1071-1077.

48. Frost, H.J. and M.F. Ashby, Deformation mechanism maps: the plasticity and creep of metals and ceramics. 1982: Pergamon press.

49. Ashby, M.F., et al., Micromechanisms of flow and fracture, and their relevance to the rheology of the upper mantle. Philosophical Transactions for the Royal Society of London. Series A, Mathematical and Physical Sciences, 1978: p. 59-95.

50. Tingdong, X., Creating and destroying vacancies in solids and non-equilibrium grainboundary segregation. Philosophical Magazine, 2003. 83(7): p. 889-899.

51. Lesuer, D., et al., Creep behavior of $\mathrm{Fe}-\mathrm{C}$ alloys at high temperatures and high strain rates. Materials Science and Engineering: A, 2001. 317(1): p. 101-107.

52. Samantaray, D., et al., Strain dependent rate equation to predict elevated temperature flow behavior of modified 9Cr-1Mo (P91) steel. Materials Science and Engineering: A, 2011. 528(3): p. 1071-1077.

53. Bhadeshia, H.K.D.H., Diffusion-controlled growth of ferrite plates in plain-carbon steels. Materials Science and Technology, 1985. 1(7): p. 497-504.

54. Essadiqi, E. and J.J. Jonas, Effect of deformation on the austenite-to-ferrite transformation in a plain carbon and two microalloyed steels. Metallurgical Transactions A, 1988. 19(3): p. 417-426.

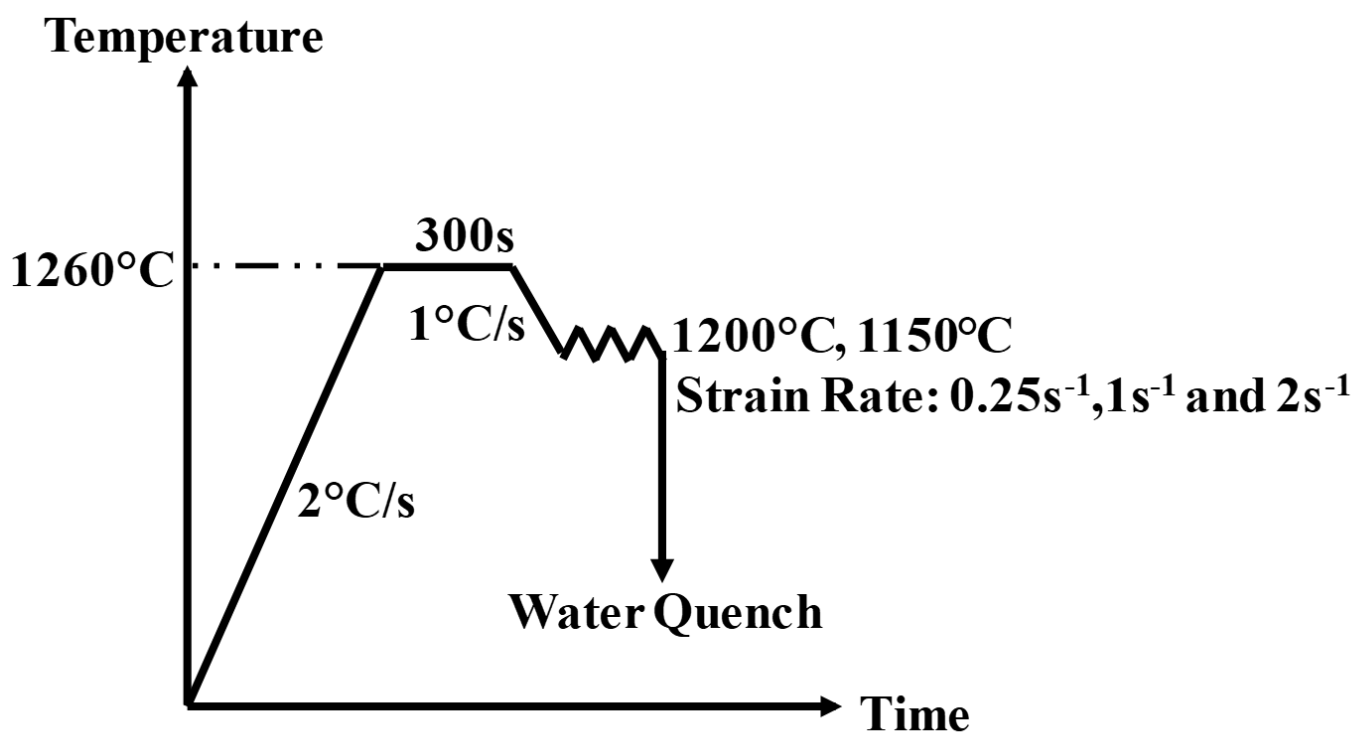


Figure 1: Schematic diagram of thermomechanical schedule for hot compression tests of as-cast medium-carbon low-alloy steel.

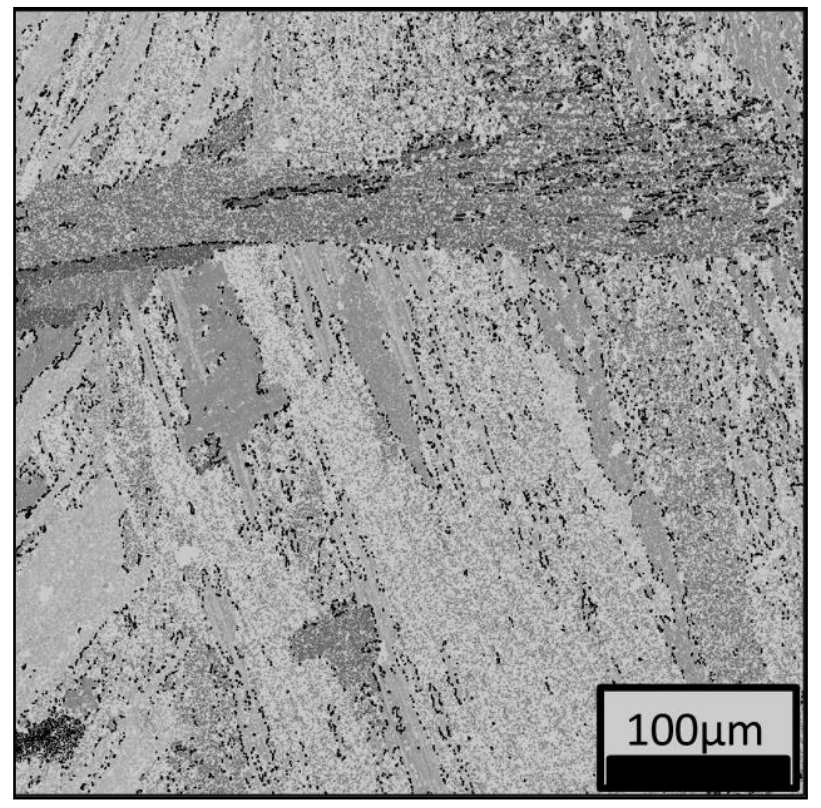

Figure 2: EBSD of initial microstructure of as-cast medium-carbon low-alloy steel in the columnar region. No specific grain boundaries can be observed in the microstructure. Black lines denote high angle grain boundaries.

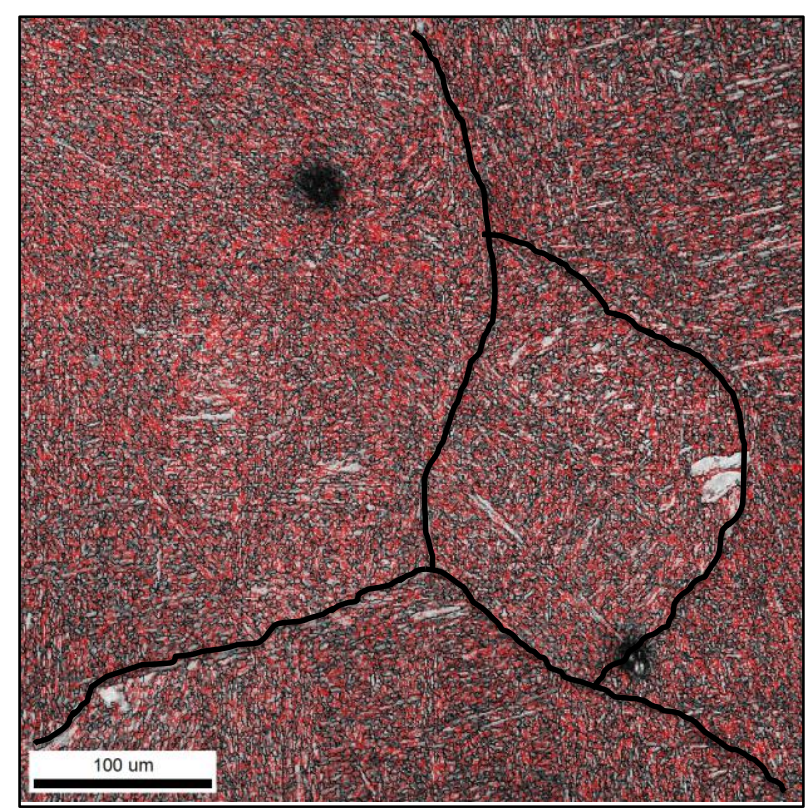


Figure 3: EBSD grain boundary map of as-cast medium-carbon low-alloy steel after austenizing at $1260{ }^{\circ} \mathrm{C}$ and then cooling to $1200{ }^{\circ} \mathrm{C}$ after which it was water quenched. Black lines denote HAGBs and red lines denote LAGBs.

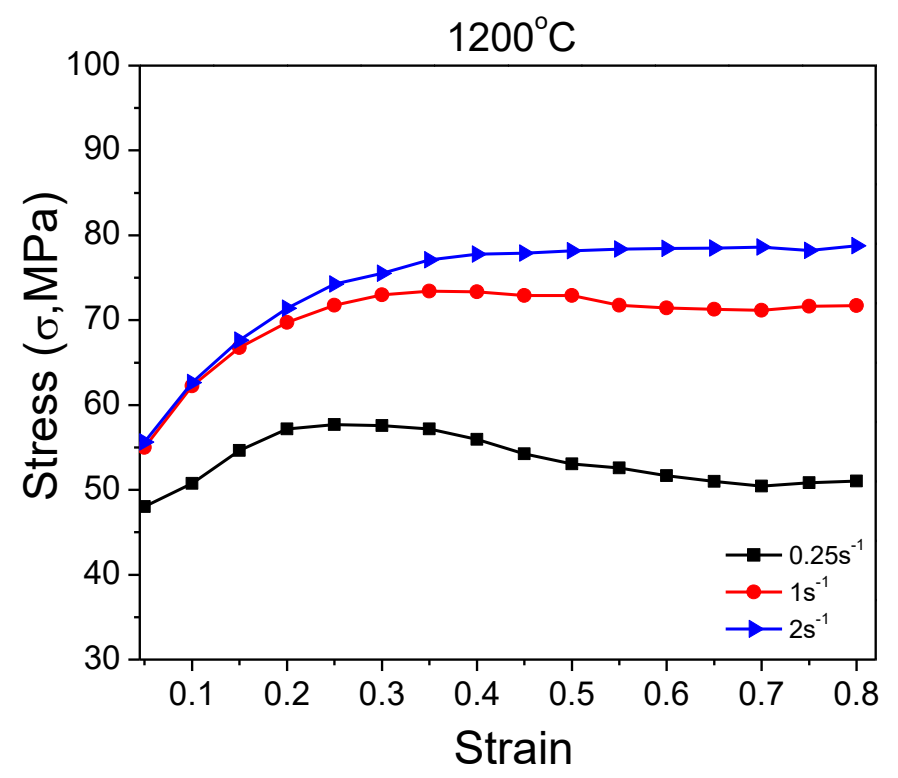

(a)

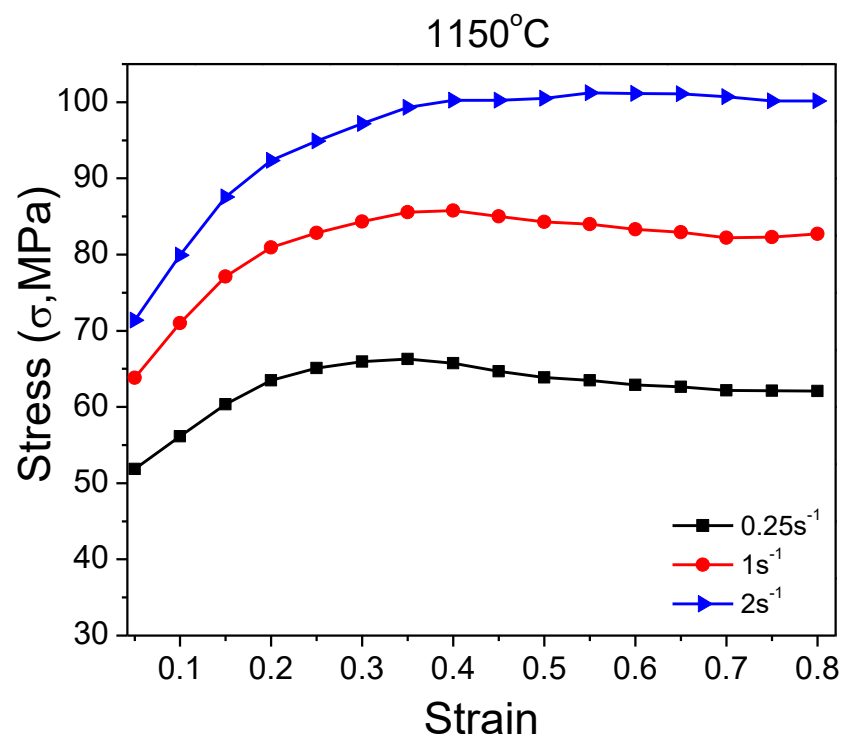

(b)

Figure 4: Stress-strain curves of as-cast medium-carbon low-alloy steel during hot isothermal compression at strain rates of $0.25 \mathrm{~s}^{-1}, 1 \mathrm{~s}^{-1}$ and $2 \mathrm{~s}^{-1}$ at deformation temperatures of (a) $1200{ }^{\circ} \mathrm{C}$, (b) $1150{ }^{\circ} \mathrm{C}$.

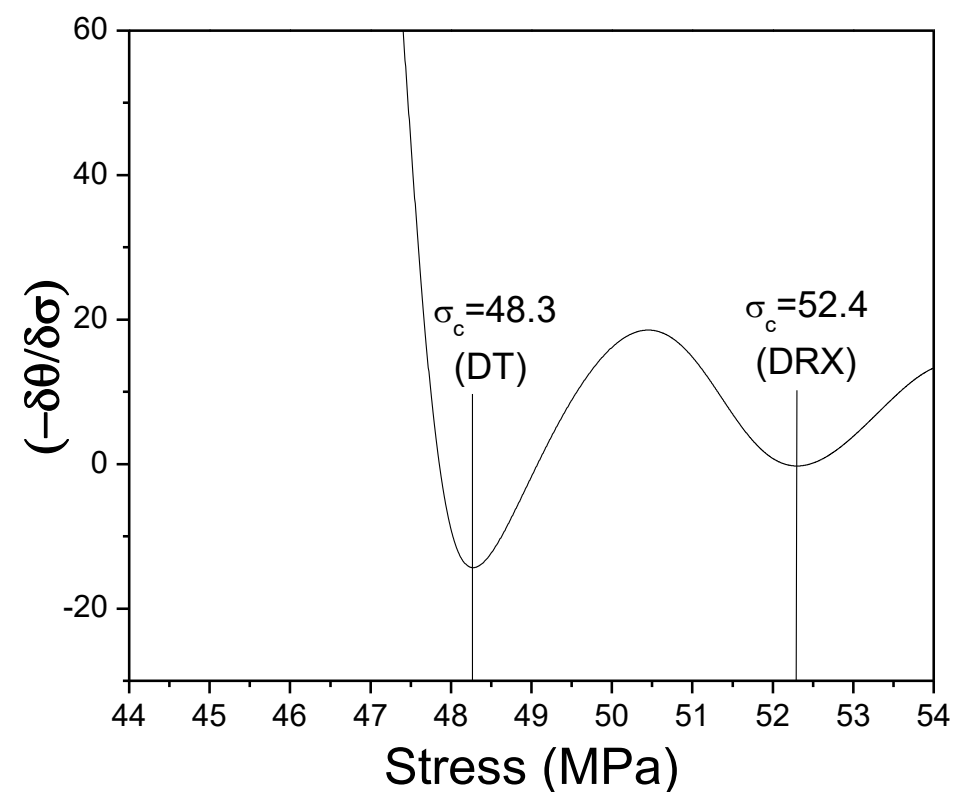


Figure 5: The double differentiation method employed in determination of the critical stresses and strains for DT and DRX. This experiment was carried out at $1200{ }^{\circ} \mathrm{C}$ and strain rate of $0.25 \mathrm{~s}^{-1}$.

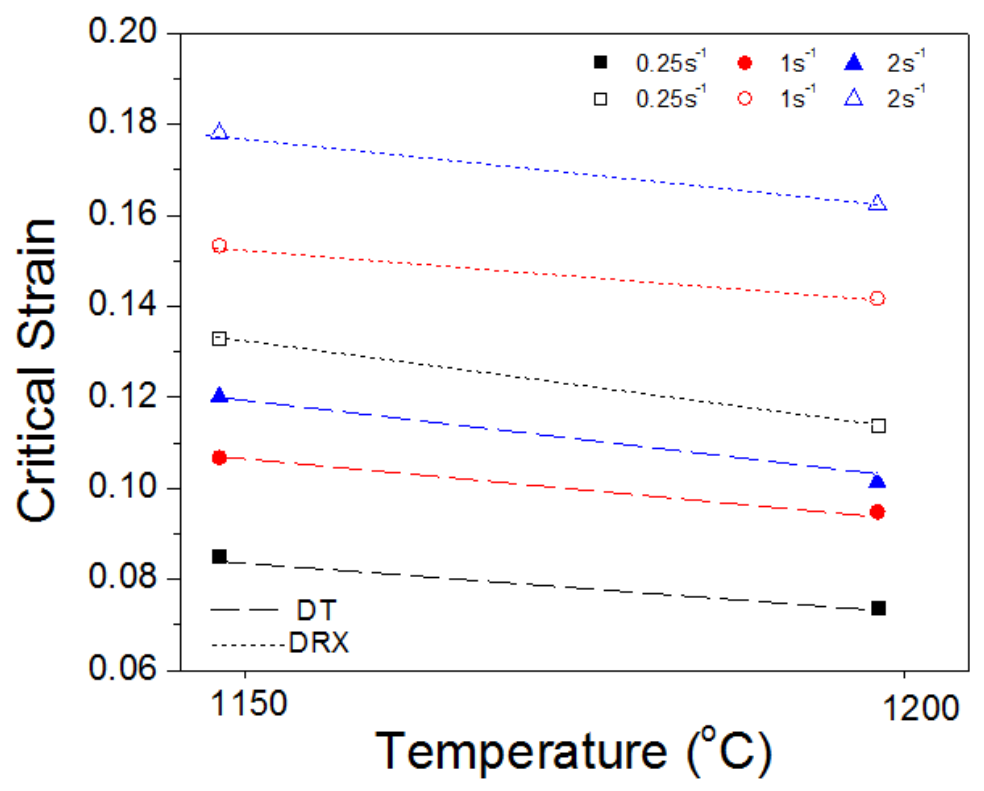

Figure 6: Critical stresses for dynamic transformation (DT) and dynamic recrystallization (DRX) determined over the temperature of $1150{ }^{\circ} \mathrm{C}$ to $1200{ }^{\circ} \mathrm{C}$ and strain rate of $0.25 \mathrm{~s}^{-1}$ to $2 \mathrm{~s}^{-1}$.
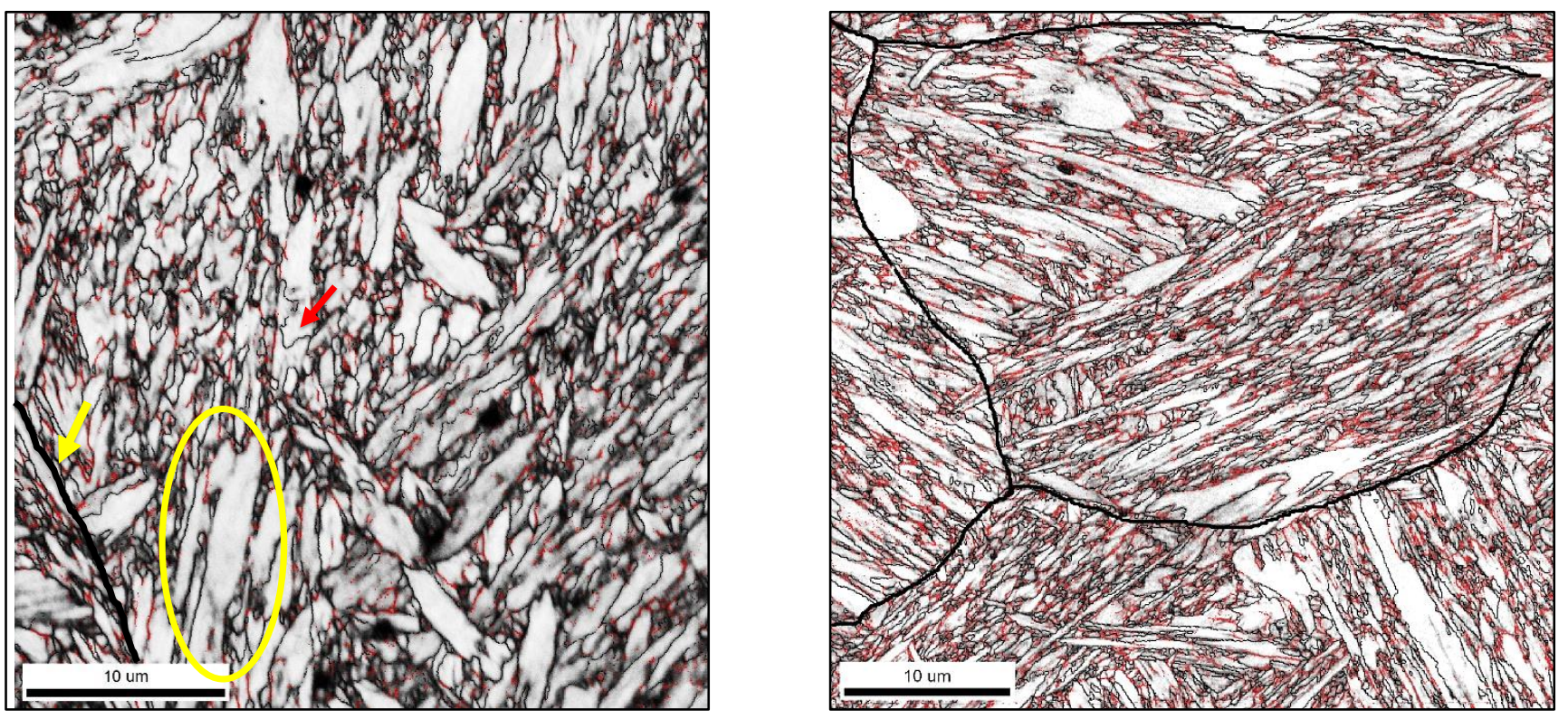
(a)

(b)

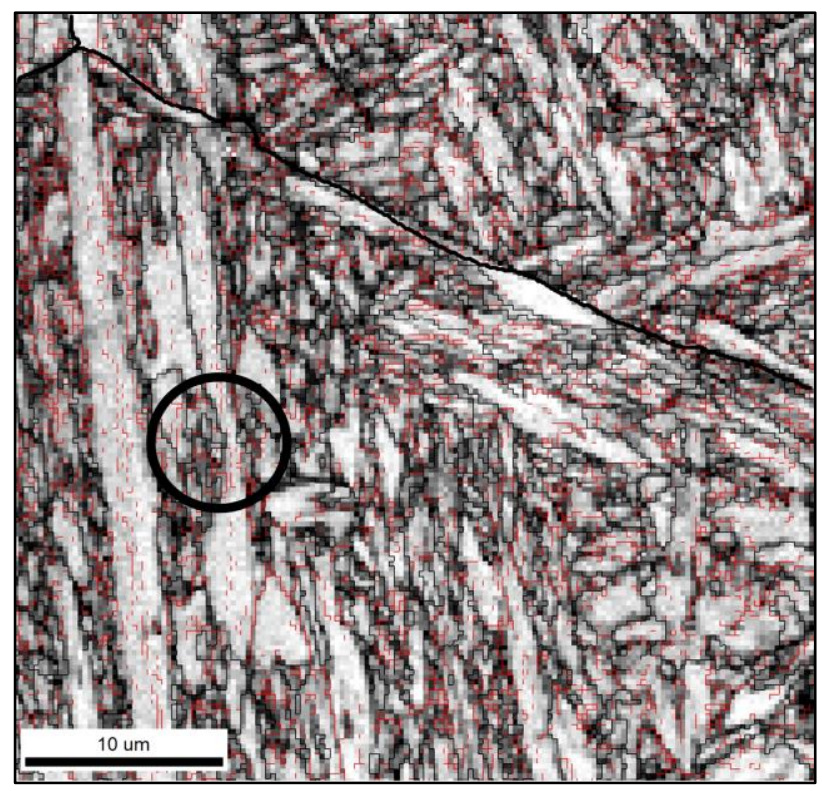

(c)

Figure 7: EBSD grain boundary map of as-cast medium-carbon low-alloy steel deformed at deformation temperature of $1200{ }^{\circ} \mathrm{C}$ at strain rate of (a) $0.25 \mathrm{~s}^{-1}$, (b) $1 \mathrm{~s}^{-1}$ and (c) $2 \mathrm{~s}^{-1}$. Black lines denote HAGB and red lines denote LAGB. 


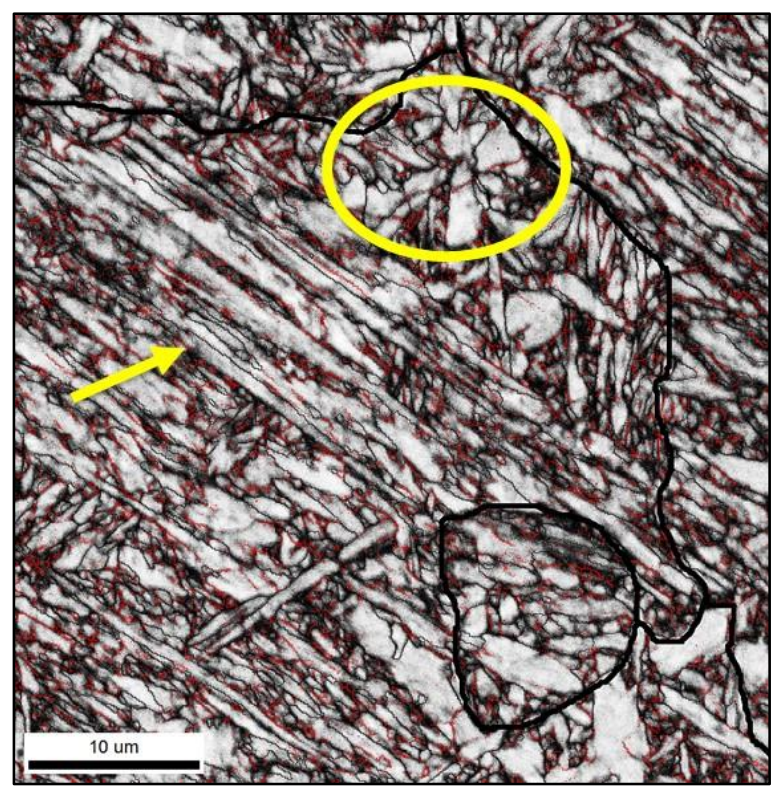

(a)

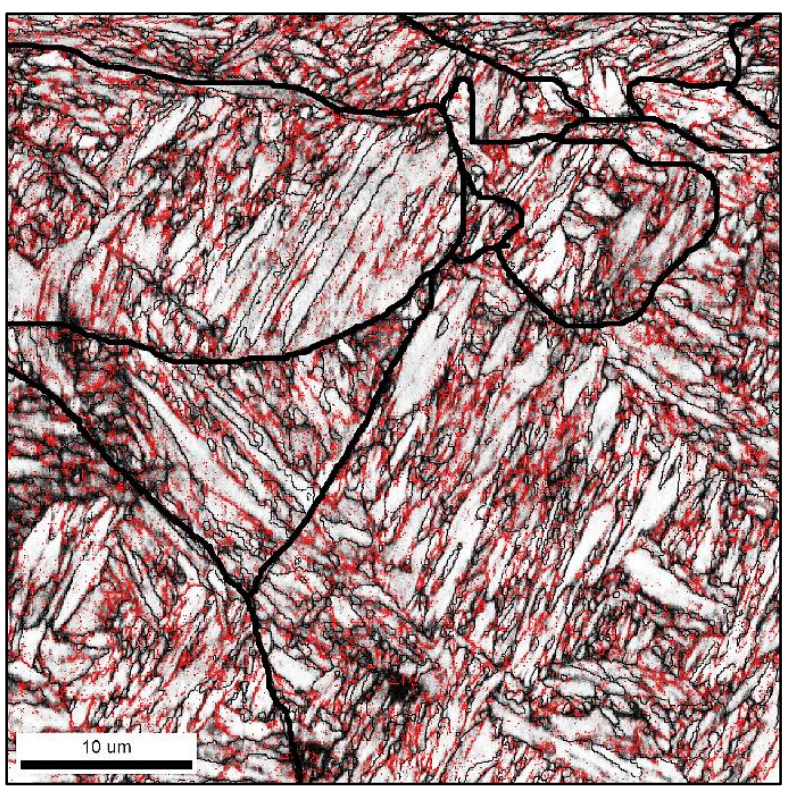

(b)

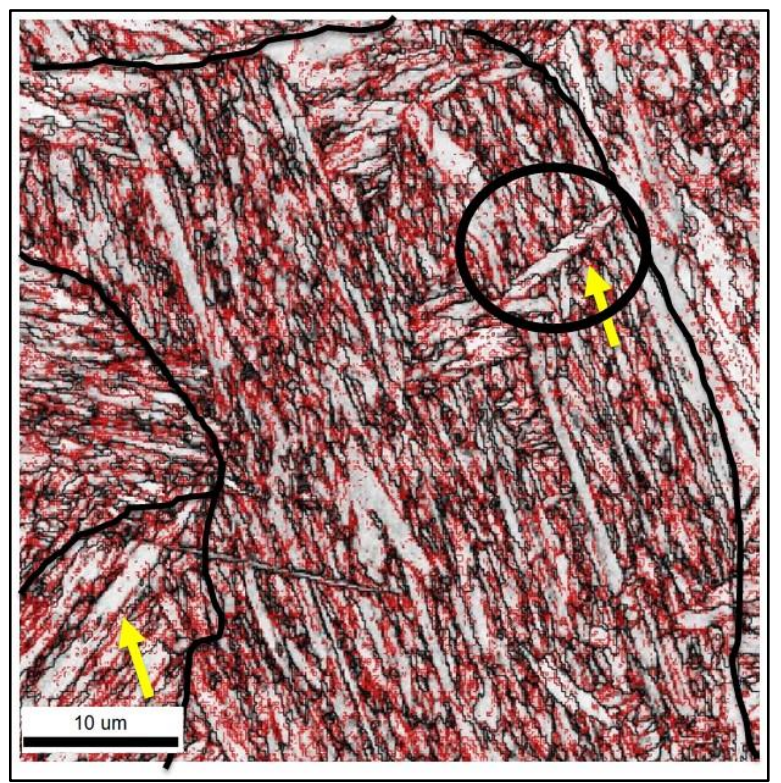

(c)

Figure 8: EBSD grain boundary map of as-cast medium-carbon low-alloy steel deformed at deformation temperature of $1150{ }^{\circ} \mathrm{C}$ and strain rate of (a) $0.25 \mathrm{~s}^{-1}$, (b) $1 \mathrm{~s}^{-1}$ and (c) $2 \mathrm{~s}^{-1}$. The black encircled region shows the disintegration of the Widmanstätten plate at high strain rate. Black lines denote HAGB and red lines denote LAGB. 


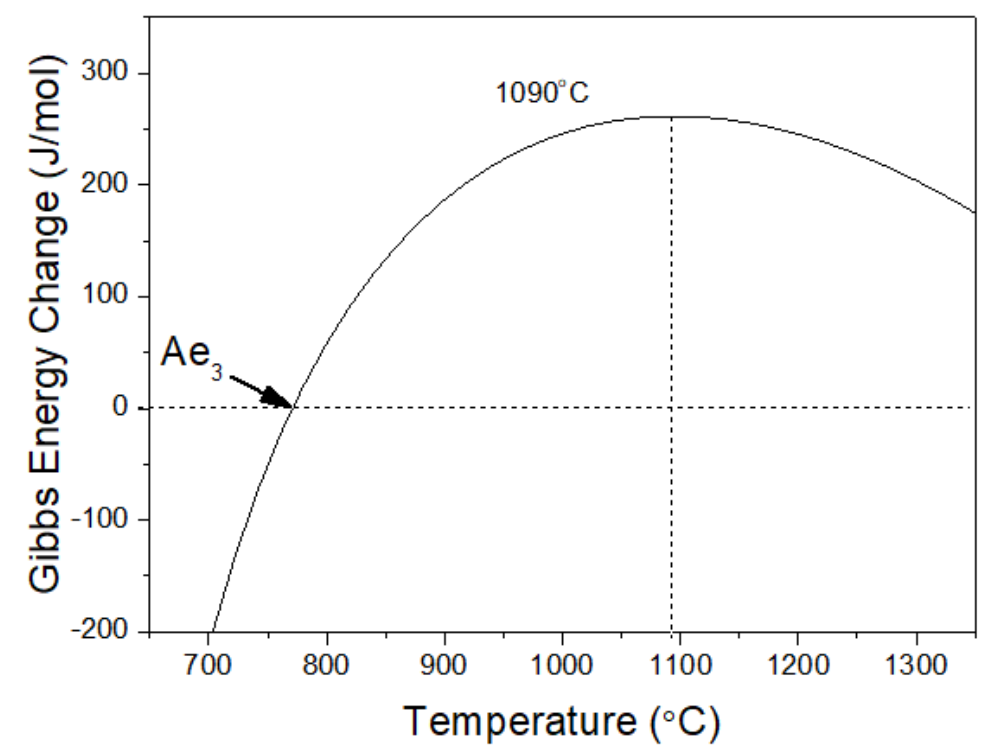

Figure $9: \Delta \boldsymbol{G}_{(\boldsymbol{\alpha}-\gamma)}$ vs T for medium-carbon low-alloy steel showing the Gibbs energy obstacle opposing dynamic transformation.

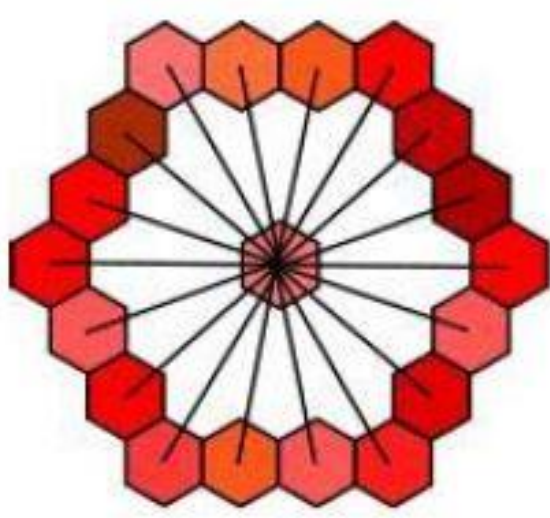

(a)

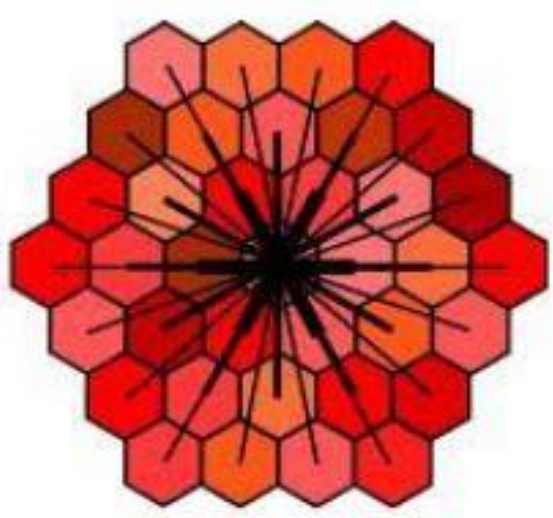

(b)

Figure 10: Schematic diagram showing (a) misorientation between a grain at the center and all other points at the perimeter of the kernel and (b) misorientation between a grain at the center and all points in the kernel. 


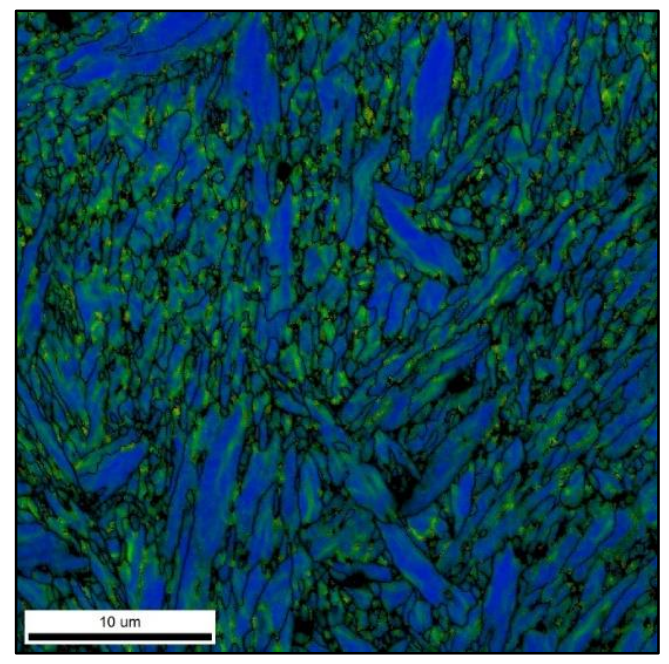

(a)

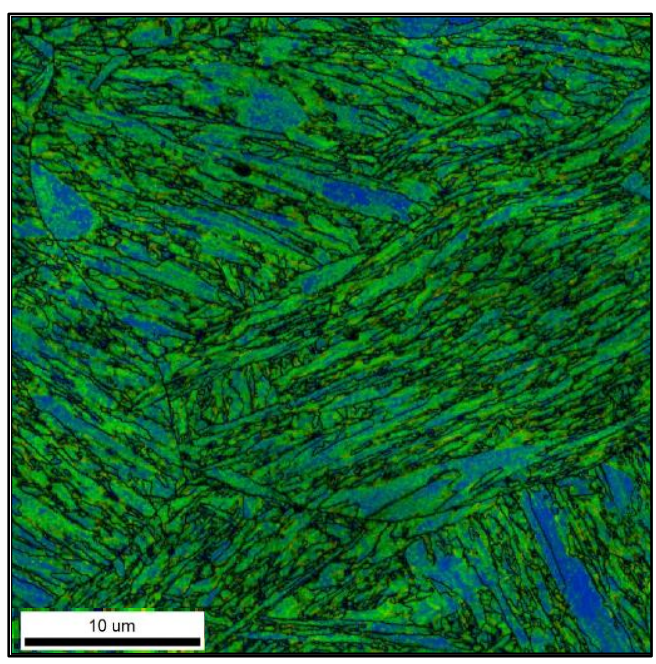

(b)

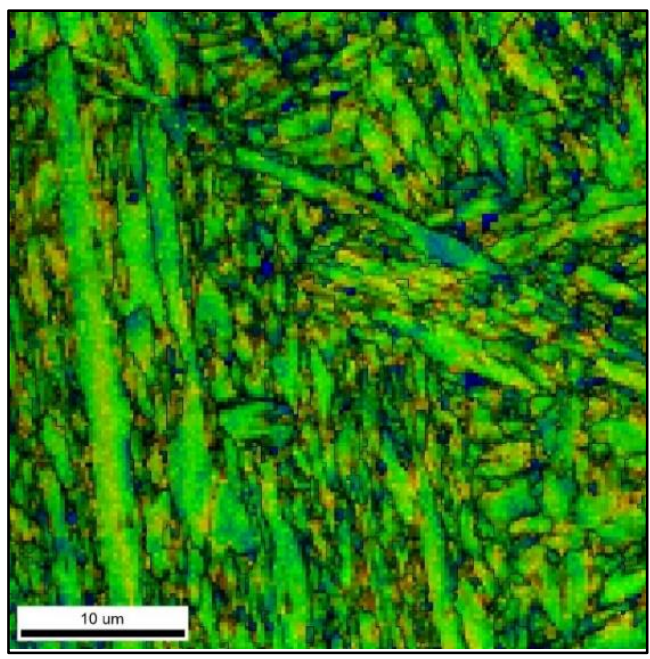

(c)

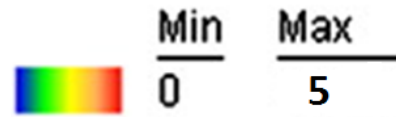

Figure 11: EBSD Kernal Average Map (KAM) of as-cast medium-carbon low-alloy steel deformed at deformation temperature of $1200{ }^{\circ} \mathrm{C}$ at strain rate of (a) $0.25 \mathrm{~s}^{-1}$, (b) $1 \mathrm{~s}^{-1}$ and (c) $2 \mathrm{~s}^{-1}$. Black lines denote HAGB. 


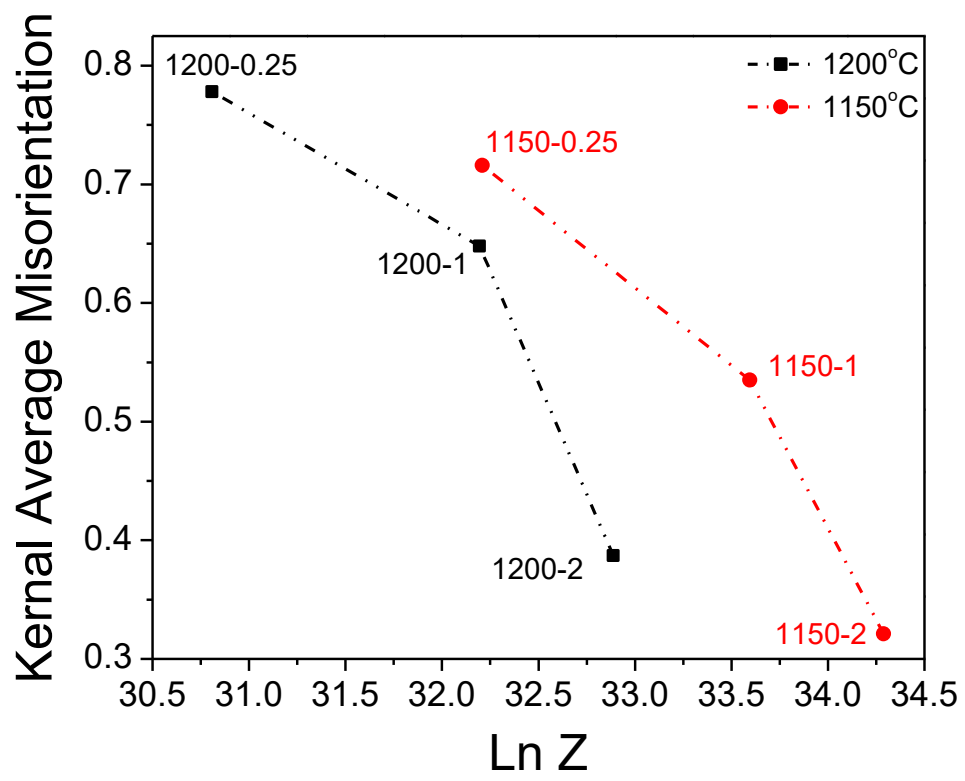

Figure 12: Graph of Kernal Average Misorientation fraction $\left(\mathrm{KAM}<2^{\circ}\right)$ vs. Ln Z. The graph reveals the effect of strain rate and temperature on ferrite fraction.

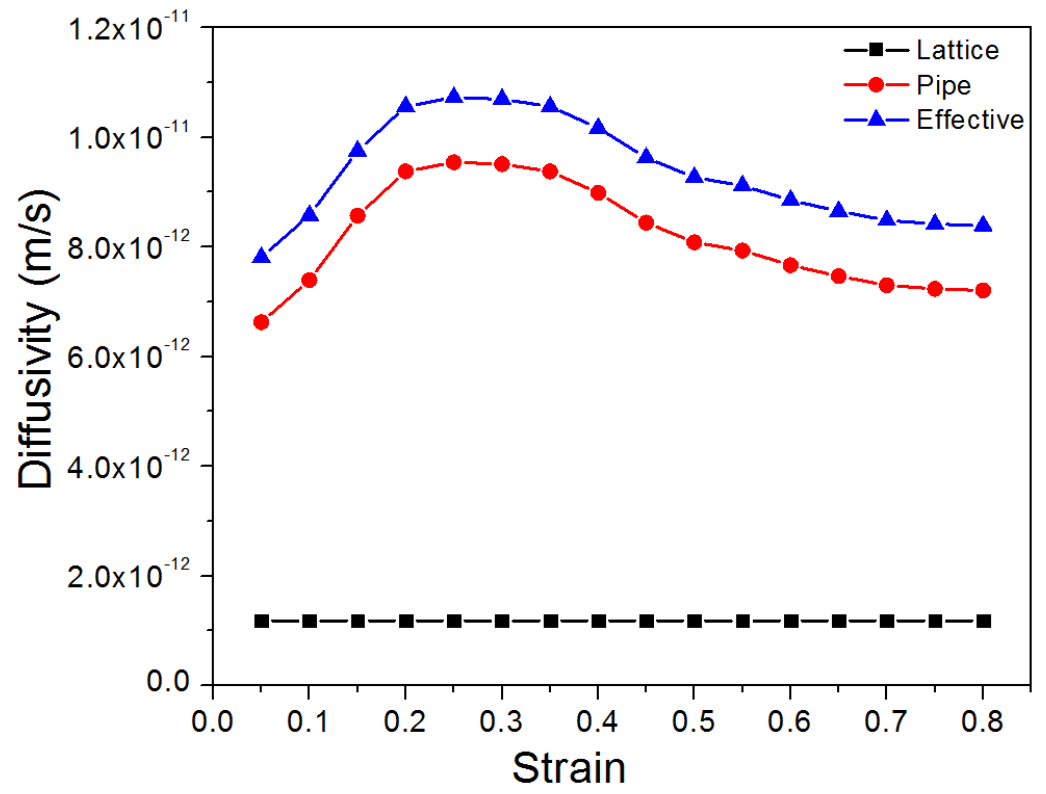

Figure 13 : Graph depicting diffusivity due to lattice diffusion, pipe diffusion and effective diffusion with regard to strain for $1200{ }^{\circ} \mathrm{C}$ and $0.25 \mathrm{~s}^{-1}$. Due to the absence of stress, $D_{L}$ is constant throughout the strain values. 


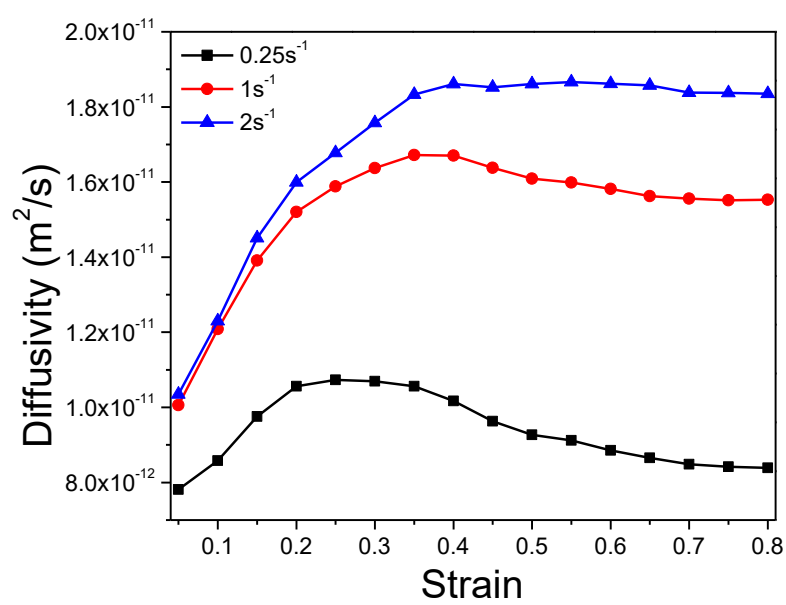

(a)

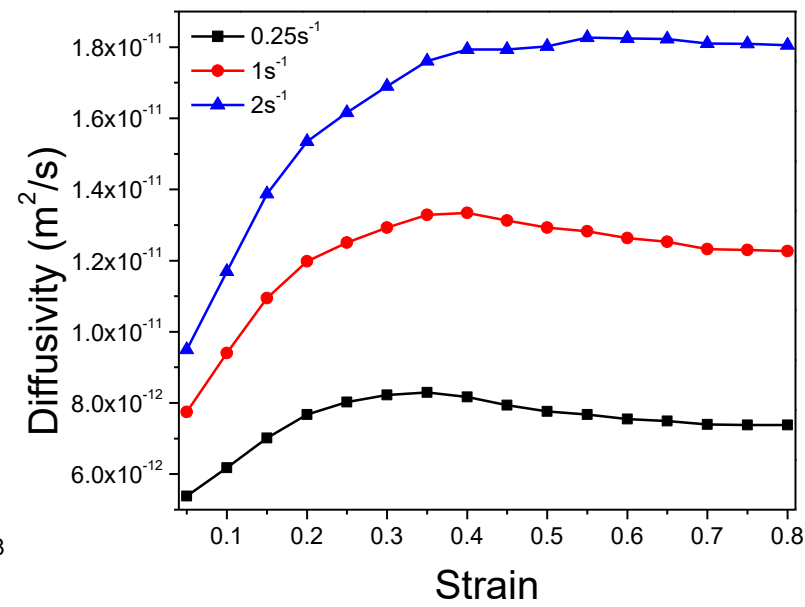

(b)

Figure 14 : Graph depicting diffusivity due to pipe diffusion vs strain for (a) $1200^{\circ} \mathrm{C}$ and (b) $1150^{\circ} \mathrm{C}$.

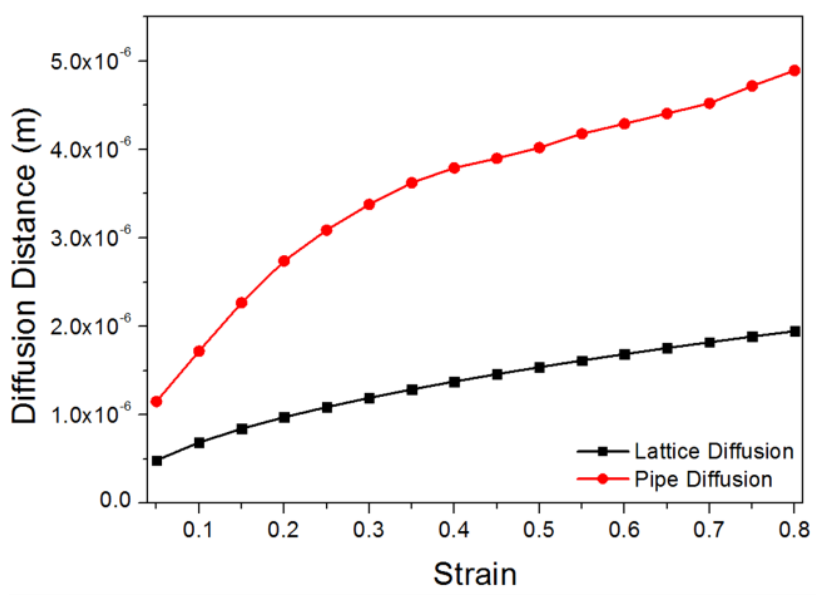

Figure 15 : Graph depicting diffusion distance of carbon atom with respect to the strain for deformation temperature of $1200^{\circ} \mathrm{C}$ and strain rate of $0.25 \mathrm{~s}^{-1}$. 


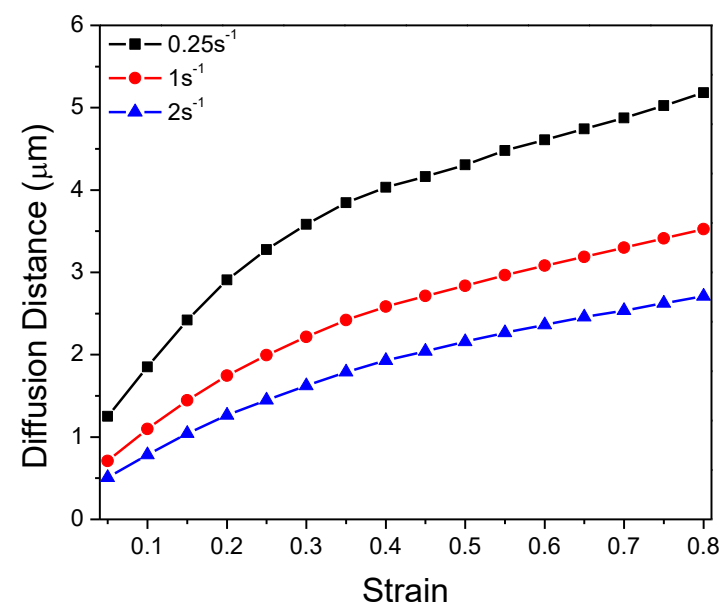

(a)

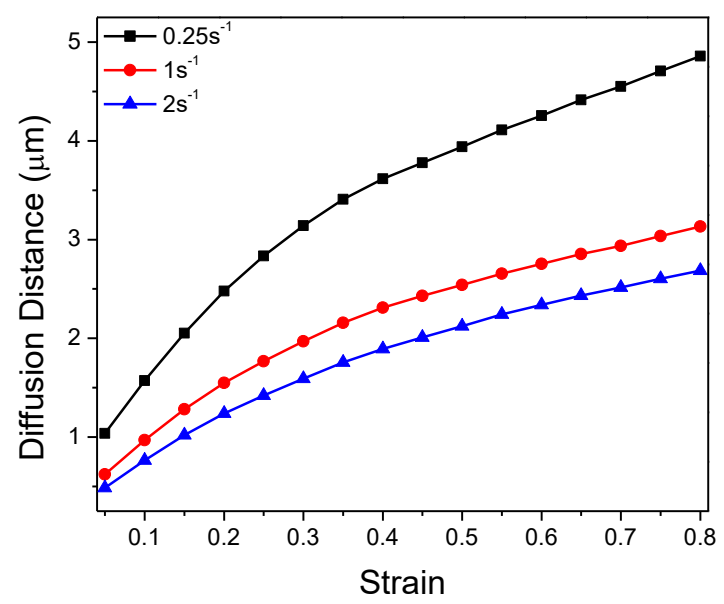

(b)

Figure 16 : Graph depicting diffusion distance of carbon w.r.t strain for (a) $1200{ }^{\circ} \mathrm{C}$ and (b) $1150^{\circ} \mathrm{C}$.

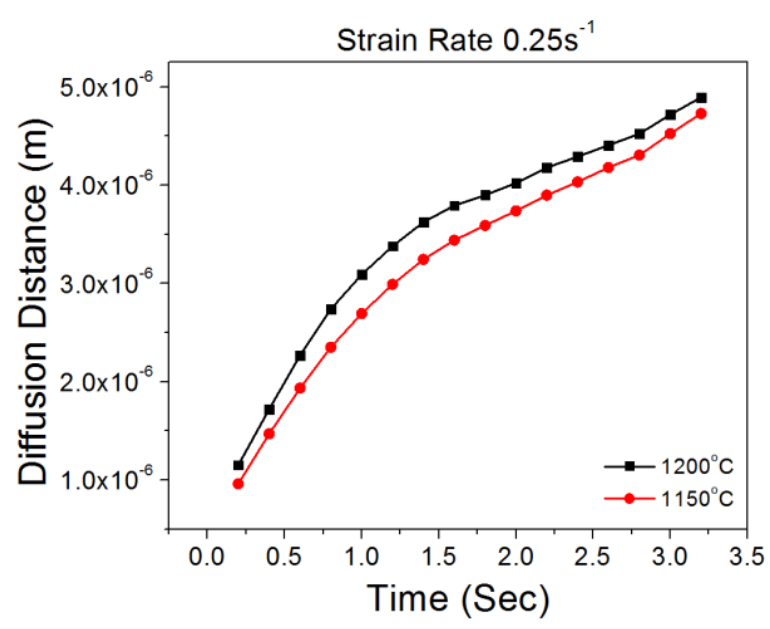

(a)

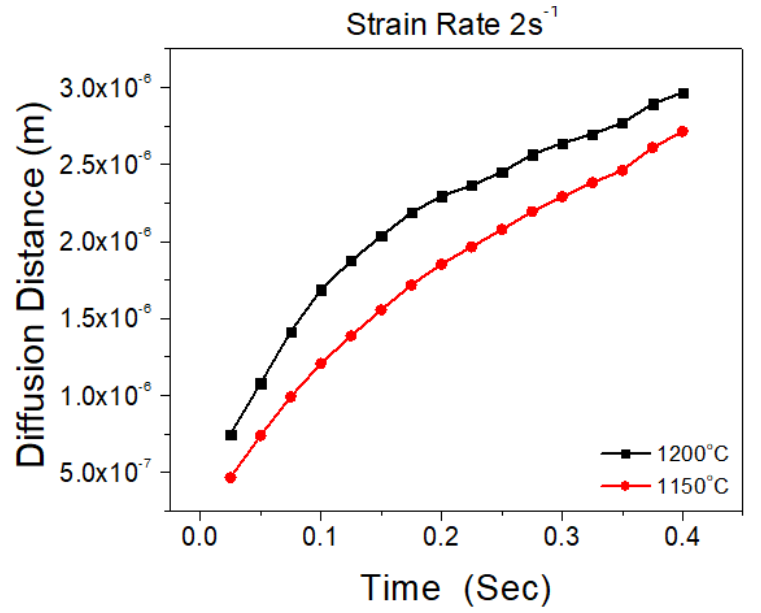

(b)

Figure 17: The variation of diffusion distance, $x(\mathrm{~m})$ due to pipe diffusion coefficient vs. deformation time, $t$ (sec) for (a) $0.25 \mathrm{~s}^{-1}$ and (b) $2 \mathrm{~s}^{-1}$. 


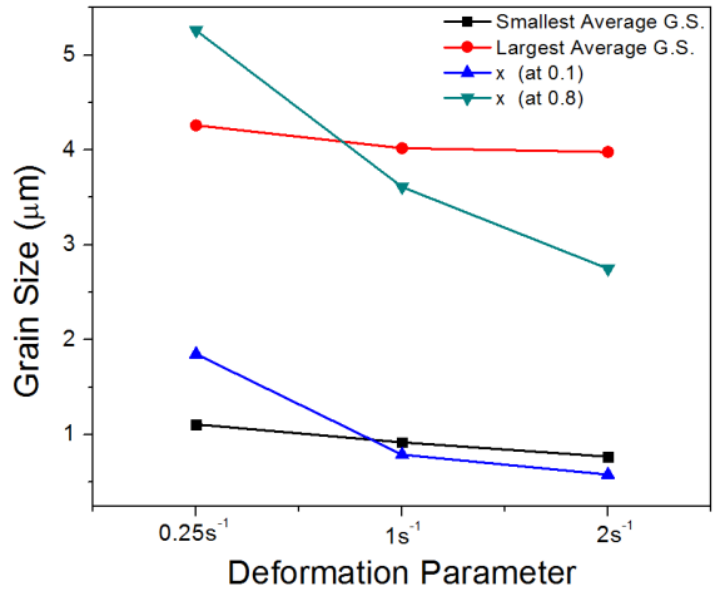

(a)

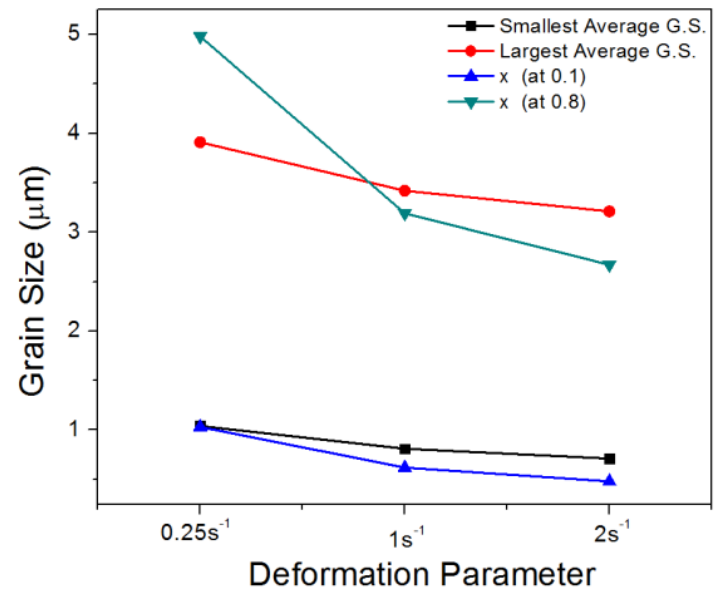

(b)

Figure 18 : Graph depicting correlation of grain size and diffusion distance w.r.t the strain rate for

(a) $1200{ }^{\circ} \mathrm{C}$ and (b) $1150{ }^{\circ} \mathrm{C}$. 


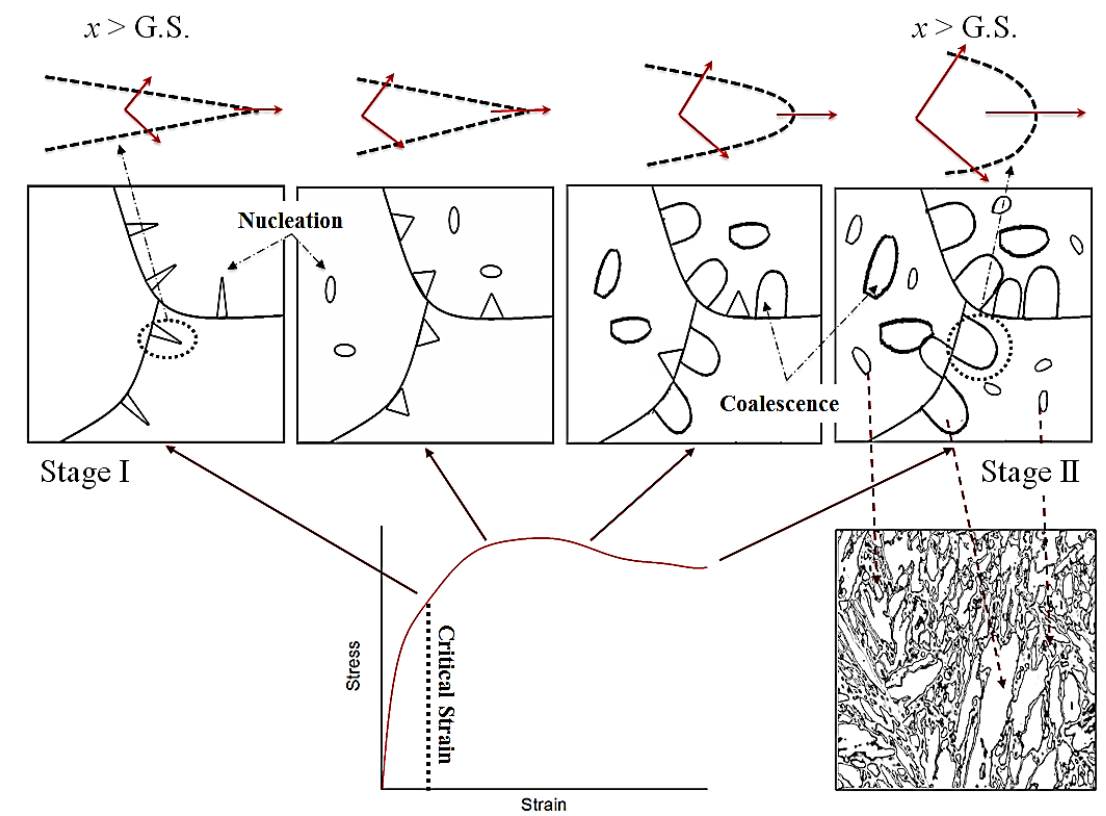

(a)

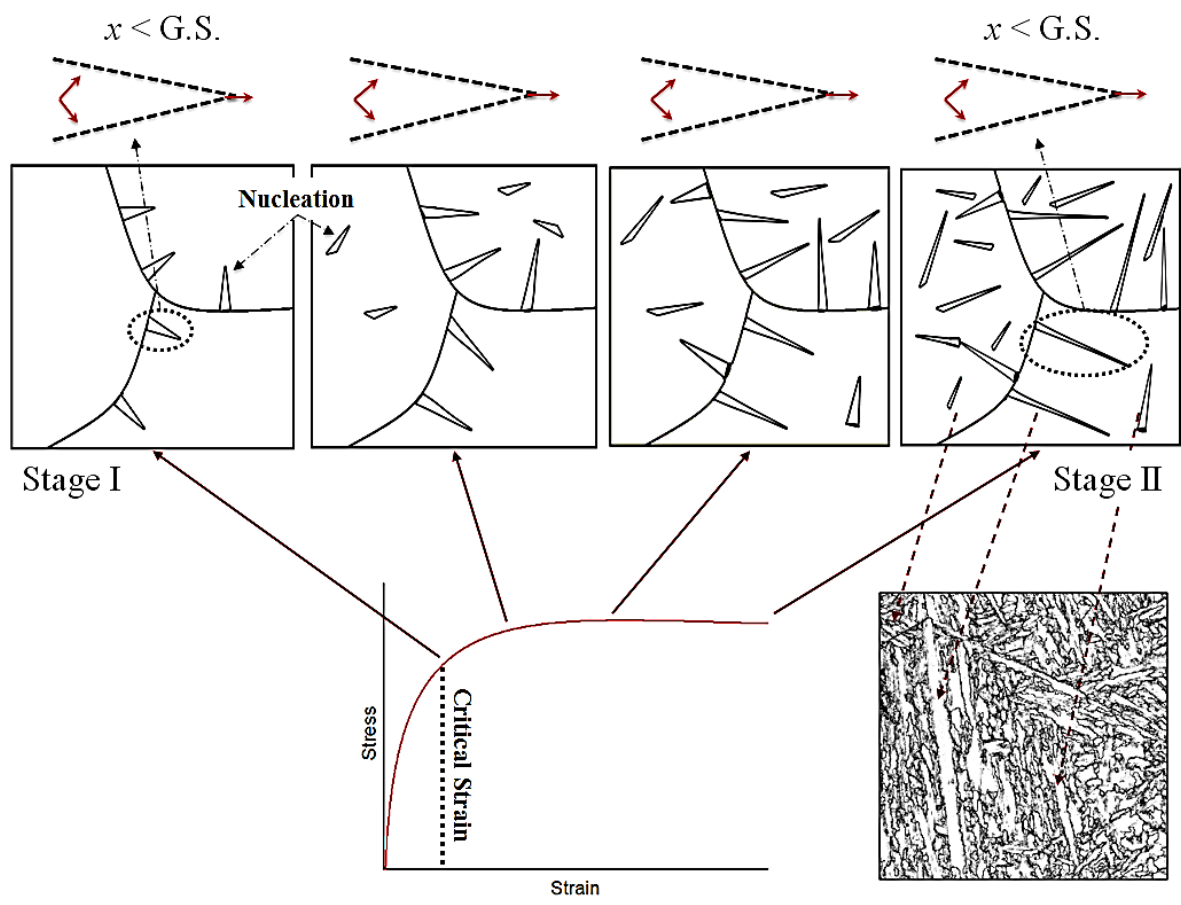

(b)

Figure 19: Schematic depicting the hypothesis to relate G.S. (grain size) and $x$ (diffusion distance of carbon) for the strain rate of (a) $0.25 \mathrm{~s}^{-1}$ and (b) $2 \mathrm{~s}^{-1}$. Red arrows represent diffusion of carbon atoms from the Widmanstätten grains (dashed lines). 
Table 1: Composition of as-cast medium-carbon low-alloy steel (wt. \%)

\begin{tabular}{cccccccc}
\hline C & Mn & Si & Mo & Cr & Other & $\begin{array}{c}\mathrm{Ae}_{3} \\
\text { Orthoequilibrium } \\
\text { temperature }\end{array}$ & $\begin{array}{c}\mathrm{Ae}_{3} \\
\text { Paraequilibrium } \\
\text { temperature }\end{array}$ \\
\hline 0.35 & 0.84 & 0.41 & 0.44 & 1.90 & Microalloying & $769.65^{\circ} \mathrm{C}$ & $748^{\circ} \mathrm{C}$ \\
\hline
\end{tabular}

\begin{tabular}{|c|c|c|}
\hline No. & $\begin{array}{l}\text { Table 2: Temperature, Pressure Coefficients, and Material Data } \\
\text { medium-carbon low-alloy steel }\end{array}$ & or Carbon in \\
\hline 1 & Bulk Modulus $K_{0}(\mathrm{MPa})$ [27] & $1.6 \times 10^{5}$ \\
\hline 2 & Shear Modulus $\mu_{0}(\mathrm{MPa})[27]$ & $7.6 \times 10^{4}$ \\
\hline 3 & Pressure Dependence $\frac{d \mu}{d \sigma_{s}}[33]$ & 1.8 \\
\hline 4 & Pressure Dependence $\frac{d K}{d \sigma_{s}}[33]$ & 4.8 \\
\hline 5 & Temperature Dependence $\frac{T_{M} d K}{K_{0} d T}[27]$ & 0.15 \\
\hline 6 & Temperature Dependence $\frac{T_{M} d \mu}{\mu_{0} d T}[27]$ & -0.46 \\
\hline 7 & Activation Volume $V_{p}^{*}\left(\frac{m^{3}}{m o l e}\right)[37]$ & $1.3 \times 10^{-6}$ \\
\hline 8 & Burger's Vector, $b_{0}(m)[33]$ & $2.5 \times 10^{-10}$ \\
\hline 9 & Pre-Exponential of pipe diffusion, $a_{p} D_{0 p}\left(\frac{m^{4}}{s}\right)[33,38]$ & $5.7 \times 10^{-24}$ \\
\hline 10 & Activation energy of pipe diffusion, $Q_{p 0}\left(\frac{K J}{\text { mole }}\right)[33]$ & 88.8 \\
\hline 11 & Pre-exponential of lattice diffusion for Carbon $D_{0 L}\left(\frac{m^{2}}{s}\right)[38]$ & $7.9 \times 10^{-7}$ \\
\hline 12 & Activation energy of lattice diffusion for Carbon, $Q_{L}\left(\frac{K J}{\text { mole }}\right)[38]$ & 148 \\
\hline 13 & Melting point of medium carbon low alloy steel, $T_{M}\left[K\left({ }^{\circ} \mathrm{C}\right)\right][27]$ & $1768(1495)$ \\
\hline
\end{tabular}

